\title{
Lepton flavor violation in string-inspired models
}

\author{
S. F. King* \\ Theory Division, CERN, CH-1211 Geneva 23, Switzerland
}

M. Oliveira

Department of Physics and Astronomy, University of Southampton, Southampton, SO17 1BJ, United Kingdom

(Received 15 April 1998; revised manuscript received 16 November 1998; published 25 June 1999)

Lepton flavor violation has been proposed as a significant test of supersymmetric unification. Here we show that such signals are also a generic feature of supersymmetric string unified models in which there is no simple unified gauge group. In realistic models of this kind which involve third family Yukawa unification and large values of $\tan \beta$, there are generally heavy right-handed (singlet) neutrinos of intermediate mass $M_{\nu}$, whose couplings violate lepton flavor. To illustrate these effects we calculate the rates for $\mu \rightarrow e+\gamma$ and $\tau \rightarrow \mu+\gamma$ in the minimal supersymmetric $\mathrm{SU}(4) \otimes \mathrm{SU}(2)_{L} \otimes \mathrm{SU}(2)_{R}$ model. Including only the minimum irreducible contributions, we find that both rates are enhanced relative to similar models with low $\tan \beta$, with $\tau \rightarrow \mu+\gamma$ providing a decisive test of such models in the near future. [S0556-2821(99)03213-0]

PACS number(s): 12.60.Jv

\section{INTRODUCTION}

Recently there has been much interest in lepton flavor violation (LFV) as a probe of physics beyond the standard model triggered by the observation of Barbieri and Hall [1,2] that processes such as $\mu \rightarrow e+\gamma$ might be very good low energy signals of supersymmetric (SUSY) grand unified theories (GUTs). In the standard model (SM) separate lepton numbers $L_{e}, L_{\mu}, L_{\tau}$ are exactly conserved, which explains the absence of LFV to remarkable accuracy (the present limit on the branching ratio for $\mu \rightarrow e+\gamma$ is approaching $10^{-11}$ ). Even if small neutrino masses are introduced into the standard model, thereby violating separate lepton numbers, the effect on $\mu \rightarrow e+\gamma$ is very small, since the amplitude is proportional to $\Delta m_{v}^{2} / M_{W}^{4}$ multiplied by suitable mixing angles, where $\Delta m_{v}^{2}$ is the difference in the squared masses of two neutrino species, and $M_{W}$ is the $\mathrm{W}$ boson mass. The introduction of SUSY allows the possibility of larger contributions to such processes since the soft SUSY breaking masses and couplings may violate separate lepton numbers by arbitrarily large amounts. This means that in SUSY there are in general additional diagrams which have in principle large contributions to LFV processes [3].

One way to avoid conflict with the experimental limits is to invoke some supergravity (SUGRA) theory [4] which leads to universal soft parameters at the Planck scale $M_{P}[5]$. In the absence of radiative corrections the slepton mass matrix in the basis $\tilde{e}, \tilde{e}^{c^{*}}$ would look similar to

$$
\left(\begin{array}{cc}
m_{e}^{\dagger} m_{e}+m_{3 / 2}^{2} I & m_{e}^{\dagger} A^{\dagger} \\
A m_{e} & m_{e} m_{e}^{\dagger}+m_{3 / 2}^{2} I
\end{array}\right),
$$

\footnotetext{
*On leave of absence from the Department of Physics and Astronomy, University of Southampton, Southampton, S017 1BJ, U.K.
}

where $m_{e}$ is the electron mass matrix, $I$ is the unit matrix, and $m_{3 / 2}, A$ are universal soft parameters. Clearly each $3 \times 3$ block of the slepton mass matrix becomes diagonal in the basis in which the electron mass matrix is diagonal, which implies no LFV. Any violation of universality will lead to off-diagonal elements in the $3 \times 3$ blocks of the slepton matrix in the charged lepton mass basis, which implies LFV. In the minimal supersymmetric standard model (MSSM) this result is preserved even in the presence of radiative corrections. This is because the renormalization group (RG) equations do not generate any off-diagonal elements for slepton masses in a basis in which the charged lepton Yukawa couplings are diagonal. Clearly if the universality assumption is relaxed then arbitrarily large LFV is possible in the MSSM. The observation of Barbieri and Hall is that with SUSY GUTs LFV is unavoidable, even with the assumption of universality. Part of the reason is that in GUTs, quarks and leptons share a common multiplet so that the lepton sector is contaminated by the flavor violating quarks. Without SUSY such an effect, though present, would be generally very weak as it scales with an inverse power of the scale of the unification scale $M_{\mathrm{GUT}}$. However in the presence of SUSY the RG running of the slepton masses between $M_{P}$ and $M_{\mathrm{GUT}}$ causes the LFV to be imprinted onto the slepton masses, which are no longer diagonal in the basis in which the leptons are diagonal. Below the GUT scale the MSSM RGEs then ensure that the LFV effect is preserved down to the $\mathrm{TeV}$ scale where it may lead to sizeable contributions to physical processes.

Although LFV can be interpreted as a signal of SUSY GUTs such as $\mathrm{SU}(5), \mathrm{SO}(10)$ [1,2] similar effects can be achieved without the presence of a GUT gauge group, even assuming strictly universal soft parameters at $M_{P}$. For example, simply adding a right-handed neutrino to the MSSM $(\operatorname{MSSM}+\nu)[6]$ will generate LFV effects due to the fact that neutrino (Dirac) Yukawa couplings are not diagonal in the basis in which the charged lepton Yukawa couplings are di- 
agonal. In the charged lepton mass basis the nondiagonal neutrino Yukawa matrix will generate off-diagonal slepton masses due to the RG running of the slepton mass matrix between $M_{P}$ and the scale $M_{\nu}$, where $M_{\nu}$ is the Majorana mass scale of the right-handed neutrinos. This will result in low energy LFV effects rather similar to those in SUSY GUTs, but without any underlying GUT gauge group. However the MSSM $+\nu$ theory is rather unconstrained compared to SUSY GUTs, and it is of interest to see if similar effects could occur in other better motivated, but non-GUT models. In particular we have in mind string-inspired models which do not involve a simple gauge group, but where the gauge couplings are unified at the string scale.

In this paper we shall focus on a particular recently proposed string-inspired model, the minimal supersymmetric $\mathrm{SU}(4) \otimes \mathrm{SU}(2)_{L} \otimes \mathrm{SU}(2)_{R}$ model [7] (see also Refs. [8,9]). In this model, quarks and leptons are unified into common multiplets, but there is no simple GUT gauge group. Instead the gauge couplings are unified with gravity at the string scale. In the minimal version [7] the only source of LFV in the 422 model is via the right-handed neutrino couplings, as in the MSSM $+\nu$ model. However, unlike the MSSM $+\nu$ model, the minimal 422 model is much more highly constrained. For example in minimal 422 there is complete Yukawa unification for the third family top, bottom, tau and tau-neutrino Yukawa couplings, which automatically leads to the prediction of a large ratio of Higgs vacuum expectation values (VEVs) with $\tan \beta$ in the range 30-60 [10] which lies beyond the scope of the results presented in Ref. [6]. As discussed in Ref. [11] the large $\tan \beta$ region involves some new effects which were neglected in previous treatments, and we are careful to include all relevant effects here.

It is worth comparing the minimal string inspired $\mathrm{SU}(4)$ $\otimes \mathrm{SU}(2)_{L} \otimes \mathrm{SU}(2)_{R}$ model to $\mathrm{SO}(10)$, which also may have Yukawa unification. In $\mathrm{SO}(10)$ color triplets with couplings to fermions are inevitably present in the effective theory beneath $M_{P}$. Indeed in SU(5) this is the primary source of LFV. But in minimal 422 such color triplets, although generically present, do not couple to fermions, and play no role in LFV. ${ }^{1}$ In general it is easy to introduce new sources of LFV, for example via LFV soft mass terms which for example may be controlled by additional $\mathrm{U}(1)_{X}$ gauged family symmetries [12]. Our approach here is to consider the minimum irreducible $e^{2}$ amount of LFV associated with this class of model. Such an approach allows unavoidable constraints to be placed on the model from the experimental limits on LFV.

Concerning our results, we find that the diagrams involving sneutrinos and charginos in the loop are found to give the

\footnotetext{
${ }^{1}$ In a nonminimal 422 model this source of flavor violation could be included at the cost of introducing eighteen unconstrained new parameters.

${ }^{2}$ It is possible that, by adding other sources of LFV, we could end up with a smaller LFV rate. Although this would be unlikely in general, it is nevertheless possible. The term minimum irreducible therefore strictly refers to the mechanism rather than the rate itself, and should be understood in this sense.
}

dominant contribution to $\mu \rightarrow e+\gamma$ and $\tau \rightarrow \mu+\gamma$. The offdiagonal sneutrino masses, which are responsible for LFV, receive contributions from the RGE evolution in the high energy region between the Planck scale and the right-handed neutrino mass scale. In the minimal supersymmetric SU(4) $\otimes \mathrm{SU}(2)_{L} \otimes \mathrm{SU}(2)_{R}$ model, with realistic masses and mixing angles, we find that generally the rates are enhanced relative to the low $\tan \beta$ case due to increased $1-2$ and $2-3$ family mixing effects. In particular we find that the predicted rate for $\tau \rightarrow \mu+\gamma$ is competitive with the current experimental limit.

The organization of the rest of the paper is as follows. In Sec. II we review the minimal 422 model. In Sec. III we describe in detail how the model was implemented giving particular emphasis to boundary conditions of the RGEs. Section IV is devoted to a detailed analysis of the one-loop decay $\mu \rightarrow e+\gamma$ and $\tau \rightarrow \mu+\gamma$. Section V contains our conclusions.

\section{THE MODEL}

Above $M_{U}$, the scale at which the three gauge couplings meet, ${ }^{3}$ we have adopted the following unified gauge group [8]:

$$
G_{U}=\mathrm{SU}(4) \otimes \mathrm{SU}(2)_{L} \otimes \mathrm{SU}(2)_{R}
$$

Here we briefly summarize the parts that are relevant for our analysis. For a more complete discussion see Ref. [9]. The left-handed quarks and leptons are accommodated in the following $F=(4,2,1), F^{c}=(\overline{4}, 1, \overline{2})$ representations:

$$
F_{i}=\left(\begin{array}{cccc}
u^{r} & u^{b} & u^{g} & \nu \\
d^{r} & d^{b} & d^{g} & e
\end{array}\right)_{i}, \quad F_{j}^{c}=\left(\begin{array}{llll}
d_{r}^{c} & d_{b}^{c} & d_{g}^{c} & e^{c} \\
u_{r}^{c} & u_{b}^{c} & u_{g}^{c} & \nu^{c}
\end{array}\right)_{j},
$$

where $i, j=1 \ldots 3$ are family indices. The MSSM Higgs fields are contained in $h=(1, \overline{2}, 2)$ :

$$
h=\left(\begin{array}{cc}
H_{u}^{0} & H_{d}^{+} \\
H_{u}^{-} & H_{d}^{0}
\end{array}\right)
$$

whereas the heavy Higgs bosons $H=(4,1,2)$ and $H^{c}$ $=(\overline{4}, 1, \overline{2})$ are denoted:

\footnotetext{
${ }^{3}$ Note that with this definition of $M_{U}$ we have $M_{U}=M_{G U T}$ $\sim 2 \times 10^{16} \mathrm{GeV}$ even though we are not considering a unified simple group.
} 


$$
\begin{gathered}
H=\left(\begin{array}{llll}
H_{u^{r}} & H_{u^{b}} & H_{u^{g}} & H_{\nu} \\
H_{d^{r}} & H_{d^{b}} & H_{d^{g}} & H_{e}
\end{array}\right), \\
H^{c}=\left(\begin{array}{llll}
H_{u_{r}^{c}} & H_{u_{b}^{c}} & H_{u_{g}^{c}} & H_{\nu^{c}} \\
H_{d_{r}^{c}} & H_{d_{b}^{c}} & H_{d_{g}^{c}} & H_{e^{c}}
\end{array}\right) .
\end{gathered}
$$

In addition to the Higgs fields in Eqs. (4) and (5) the model also involves an $\mathrm{SU}(4)$ sextet field $D=(6,1,1)=\left(D_{3}, D_{3}^{c}\right)$.

The superpotential of the minimal 422 model is [7]

$$
\begin{aligned}
\mathcal{W}= & S\left[\kappa\left(H^{c} H-M_{P}^{2}\right)+\lambda h^{2}\right]+\lambda_{H} D H H+\lambda_{H^{c}} D H^{c} H^{c} \\
& +\lambda_{33} F^{c}{ }_{3} F_{3} h+\lambda_{i j} F_{i}^{c} F_{j} h \frac{\left(H^{c} H\right)^{n}}{M_{P}^{2 n}}+\lambda_{\nu^{c} i j} F_{i}^{c} F^{c}{ }_{j} \frac{H H}{M_{P}},
\end{aligned}
$$

where $S$ denotes a gauge singlet superfield, the parameters $\kappa, \lambda$ are taken to be real and positive, and $h^{2}$ denotes the unique bilinear invariant $\epsilon^{i j} h_{i}^{(1)} h_{j}^{(2)}$. Also, $M_{P}(\simeq 2.4$ $\left.\times 10^{18} \mathrm{GeV}\right)$ denotes the "reduced" Planck mass. As a result of the superpotential terms involving the singlet $S$ the Higgs fields develop VEVs, $\langle H\rangle=\left\langle H_{\nu}\right\rangle \sim M_{U}$ and $\left\langle H^{c}\right\rangle$ $=\left\langle H_{\nu^{c}}\right\rangle \sim M_{U}$, which lead to the symmetry breaking

$$
\mathrm{SU}(4) \otimes \mathrm{SU}(2)_{L} \otimes \mathrm{SU}(2)_{R} \rightarrow \mathrm{SU}(3)_{c} \otimes \mathrm{SU}(2)_{L} \otimes \mathrm{U}(1)_{Y}
$$

The singlet $S$ itself also naturally develops a small VEV of order the SUSY breaking scale [7] so that the $\lambda S$ term in Eq. (6) gives an effective $\mu$ parameter of the correct order of magnitude. Under Eq. (7) the Higgs field $h$ in Eq. (4) splits into the familiar MSSM doublets $H_{u}$ and $H_{d}$ whose neutral components subsequently develop weak scale VEVs $H_{u}^{0}$ $=\left\langle v_{u}\right\rangle$ and $H_{d}^{0}=\left\langle v_{d}\right\rangle$ with $\tan \beta=v_{u} / v_{d}$.

This model has Yukawa unification for the third family $[13,14]$ which leads to a large top mass $m_{\text {top }}>165 \mathrm{GeV}$ and $\tan \beta \sim m_{\text {top }} / m_{\text {bottom }}$. First and second family Yukawa couplings are effectively generated by nonrenormalizable operators which are suppressed by powers of a heavy scale $M_{P}$ $>M_{U}$. In the 422 model, these operators can be constructed from different theoretical group contractions of the fields such as $[15,16]$

$$
\mathcal{O}_{i j}=F_{i}^{c} \lambda_{i j} F_{j} h\left(\frac{H H^{c}}{M_{P}^{2}}\right)+\text { H.c. }
$$

The idea is that when the heavy Higgs bosons develop their large VEVs such operators reduce to effective Yukawa couplings of the form $F^{c} \lambda F$ with a small $\left(M_{U}^{2} / M_{P}^{2}\right)$ coefficient. Assuming a (well motivated) texture [17] for the Yukawa matrix at $M_{U}$ and suitably choosing a set of operators, suc- cessful predictions can be made for some SM parameters. Vertical splittings within a particular family are accounted for by group theoretical Clebsch factors [15]. A detailed analysis of this approach for the 422 model can be found in Ref. [18]. The nonrenormalizable operators involving the right-handed neutrino result in Majorana masses of the form $1 / 2 M_{\nu} \nu^{c} \nu^{c}$, where $M_{\nu} \sim M_{U}^{2} / M_{P}$, which enables righthanded neutrinos to decouple at the scale $M_{\nu}$, leading to a Gell-Mann-Ramond-Slansky seesaw mechanism.

The $D$ field does not develop a VEV but the terms $H H D$ and $H^{c} H^{c} D$ combine the color triplets parts of $H, H^{c}$ and $D$ into acceptable GUT scale mass terms [9]. We note that the 422 symmetry also allows the couplings

$$
\begin{gathered}
F F D \rightarrow Q Q D_{3}+Q L D_{3}^{c}, \\
F^{c} F^{c} D \rightarrow u^{c} d^{c} D_{3}^{c}+u^{c} e^{c} D_{3}+d^{c} \nu^{c} D_{3},
\end{gathered}
$$

which obviously would generate additional LFV signals. However these may be forbidden by a global R-symmetry [7]. Their exclusion here is in keeping with the general philosophy of the approach which is to consider the minimum amount of irremovable LFV in the model, so that LFV becomes an unavoidable signal of the model.

\section{PROCEDURE}

In this section we describe how the 422 model was implemented. We considered three fundamental scales: $M_{\text {SUSY }}$ which was assumed to equal the top quark mass, $M_{U}$ $=2.0 \times 10^{16} \mathrm{GeV}$ the scale of coupling unification and $M_{P}$ $=2.4 \times 10^{18} \mathrm{GeV}$. An additional scale $M_{\nu}$ describing the energy at which the right-handed neutrinos decouple via seesaw mechanism was introduced. Cosmological constraints require $10^{10} \mathrm{GeV} \leqslant M_{\nu} \leqslant M_{U}$ [19]. Experimentally viable boundary conditions were imposed at each fundamental scale and we used one-loop matrix RGEs [20] to relate parameters at different energies (particle threshold effects were ignored).

We now turn to describe the algorithm of the program. Since we want to achieve third family Yukawa unification (3FYU) at $M_{U}$ [21], which crucially depends on the unknown low energy values of $m_{\text {top }}, m_{\nu_{\tau}}=v_{u} \lambda_{\nu_{\tau}}$ and $\tan \beta$ an iterative procedure is needed. Initial estimates for these parameters are guessed and, along with gauge couplings and all Yukawa matrices, are run, first from their definition values to $M_{\text {SUSY }}=m_{\text {top }}$ and afterwards from $M_{\text {SUSY }}$ to $M_{U}$. At $M_{U}$ the guesses are tested to see if they actually lead to $3 F Y U$. This is unlikely to happen in the first attempt therefore we induce slight changes in our initial guesses and repeat the above process again. After a few iterations it starts to be obvious that some guesses are more successful than others. These are subjected to further pertubative analysis allowing more precise GUT unification. This recursive approach is repeated many times until the condition $\lambda_{t}=\lambda_{b}=\lambda_{\nu_{\tau}}=\lambda_{\tau}$ is verified to a satisfactory accuracy (typically $1 \%$ ). At this point some comments are worth making. The running of masses to 


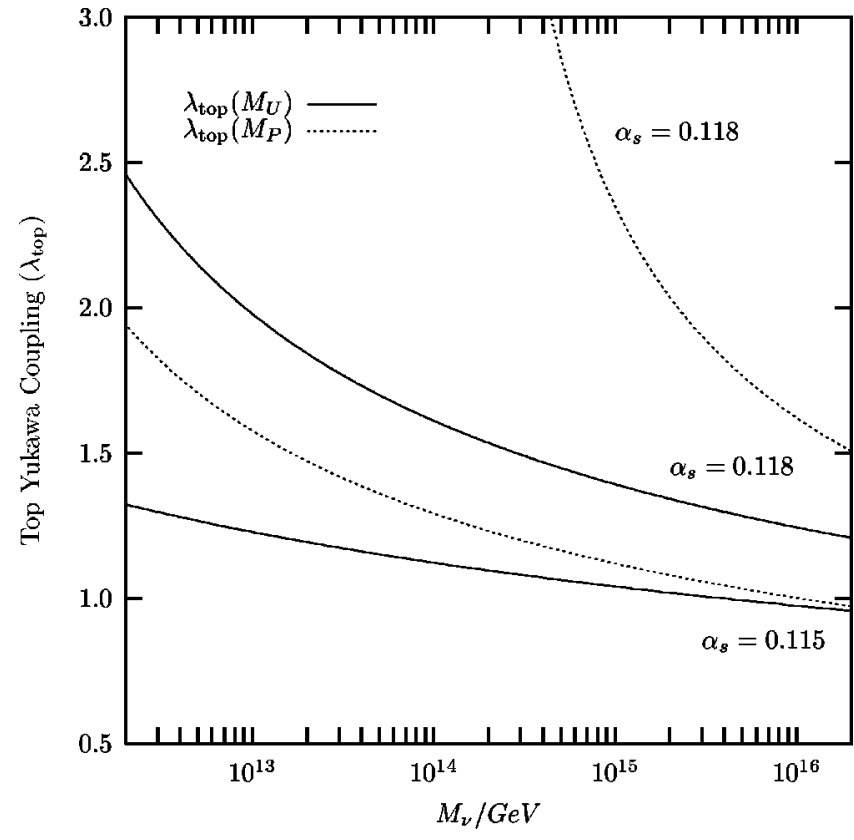

FIG. 1. Dependence of the third family unified Yukawa coupling $\lambda_{\text {top }}$ at the unification $M_{U}$ (solid) and Planck $M_{P}$ (dashed) energy with the right-handed neutrino decoupling scale $M_{\nu}$ for two values of $\alpha_{s}$. The two upper (lower) lines correspond to $\alpha_{s}$ $=0.118\left(\alpha_{s}=0.115\right)$.

$M_{\text {SUSY }}$ was done using the standard model RGEs (one-loop QED, three-loop QCD [22]) and it is necessary as it provides important mass corrections especially for light quarks. After the whole iterative process is complete we are left with predictions for our guesses based on the assumption of 3FYU. To have an idea, for the input $\alpha_{s}=0.115, m_{b}=4.25 \mathrm{GeV}$, $M_{\nu}=M_{U}$ we obtained the following values $m_{\text {top }}$ $\sim 175 \mathrm{GeV}, m_{\nu_{\tau}} \sim 122 \mathrm{GeV}$ and $\tan \beta \sim 56$. The dependence on $M_{\nu}$ is mostly felt by $m_{\nu_{\tau}}$ which can decrease to $115 \mathrm{GeV}$ when $M_{\nu} \sim 10^{12} \mathrm{GeV}$. The variations with $\alpha_{s}$ and $m_{b}$ are considerable and well documented [18]. We note that it is important to watch for the magnitude of the third family of the Yukawa couplings at $M_{P}$ because they are very sensitive to $\alpha_{s}$ and $M_{\nu}$, therefore can easily acquire values outside the range allowed in perturbative regime (Fig. 1).

At $M_{U}$, we must match all the Yukawa matrix couplings to the ones which can be obtained from nonrenormalizable operators such as Eq. (8). These new Yukawa matrices are not unique. However they are constrained by the fact that they must predict the same (known) physics as the ones they replace, i.e., both must have identical eigenvalues and quark mixing angles (for the sake of simplicity we did not consider $C P$ violating phase). Several forms of these Yukawa matrices were extensively studied in Refs. $[18,10]$ for the 422 model. Here we will only consider the following particular one: ${ }^{4}$

${ }^{4}$ Other choices would lead to same order of magnitude results.

$$
\begin{aligned}
& \lambda_{u}=\left(\begin{array}{ccc}
0 & Y^{n=3} & 0 \\
Y^{A d} & Y^{D}-Y^{C} & 0 \\
0 & Y^{B} & Y^{33}
\end{array}\right), \\
& \lambda_{d}=\left(\begin{array}{ccc}
0 & Y^{1} & 0 \\
3 Y^{A d} & -\left(Y^{D}+Y^{C}\right) & 0 \\
0 & -Y^{B} & Y^{33}
\end{array}\right), \\
& \lambda_{e}=\left(\begin{array}{ccc}
Y^{1} & 0 \\
0 & 3\left(Y^{D}+Y^{C}\right) & 0 \\
9 Y^{A d} & -Y^{B} & Y^{33} \\
0 & & \\
\lambda_{\nu} & = & \\
0 & Y^{n=3} & 0 \\
3 / 4 Y^{A d} & -3\left(Y^{D}-Y^{C}\right) & 0 \\
0 & Y^{B} & Y^{33}
\end{array}\right) .
\end{aligned}
$$

We briefly explain their form. The zeros in positions $(1,3)$, $(3,1)$ are motivated by correspondingly small entries on the quark Cabibbo-Kobayashi-Maskawa (CKM) matrix. The zero in $(2,3)$ only effects the right-handed mixing matrix (because of high family hierarchy), thus it is convenient as it improves predictability. Two operators were needed in the $(2,2)$ position because of particular high charm-muon splitting [18]. The operator $Y^{B}$ generates $V_{23} \sim V_{32}$, while $Y^{A d}$ and $Y^{1}$ generate $V_{12} \sim V_{21}$ and first family masses. ${ }^{5}$ The coefficients on different matrices associated with the same operator $Y$ are the Clebsch 422 factors mentioned in Sec. II. Solutions for the $Y \mathrm{~s}$ were numerically searched for the input: $V_{23}, m_{\text {charm }}, m_{\mu}$ and $V_{12}, m_{\text {up }}, m_{e}$, which enabled three quantities to be predicted: $V_{13}, m_{\text {down }}, m_{\text {strange }}$ (see Appendix $\mathrm{D}$ for results). Notice that this model predicts the experimentally unavailable Dirac neutrino masses $m_{\nu_{e}}, m_{\nu_{\mu}}, m_{\nu_{\tau}}$ and the lepton Dirac CKM matrix $V^{L}$. The prediction of physical neutrino masses and mixing angles relies on the knowledge of the right-handed neutrino Majorana mass matrix $M_{\nu}$. Following Ref. [10] we shall assume that $M_{\nu}$ is proportional to the unit matrix. This rather ad hoc assumption at least has the virtue that it leads to the result that the modulus of the leptonic CKM matrix elements are equal to those calculated just from the Dirac neutrino mass parts [10]. It also means that the physical neutrino masses are determined by a single mass parameter which we continue to denote by $M_{\nu}$, where this parameter henceforth refers to the overall factor multiplying the unit Majorana matrix rather

\footnotetext{
${ }^{5}$ Actually, since the $(n=2) Y^{1}$ operator has a vanishing Clebsch for the up-type fermions, we are forced to introduce a further $Y^{n=3}$ operator in the $(1,2)$ position, if we want to avoid a massless up quark.
} 
than the matrix itself. With a suitable choice of $M_{\nu}$ this simple assumption leads to a physical muon-neutrino and electron-neutrino mass spectrum suitable for the MSW solution to the solar neutrino problem, with a tau-neutrino in the correct range for hot dark matter, and with muon-tau neutrino oscillations in the observable range of the CHORUS experiment [10]. If this assumption is relaxed one would generally expect qualitatively similar effects both in the neutrino spectrum, and in the physics of LFV considered here.

After having set experimentally viable Yukawa matrices at $M_{U}$, according to the above boundary conditions, we used 422 RGEs (see Appendix B) to run them to $M_{P}$. In this high energy region we treated the theory described in Sec. II in the following effective way. To begin with, we regarded the nonrenormalizable operators as yielding four effective Yukawa matrices, whose RG evolution is described by standard RGEs appropriate to the larger gauge group SU(4) $\otimes \mathrm{SU}(2)_{L} \otimes \mathrm{SU}(2)_{R}$. The terms involving the singlet $S$ which give rise to an effective $\mu$ parameter below the scale $M_{U}$, were regarded as an effective $\mu$ parameter above this scale similar to the MSSM. Finally we allowed extra $D$ and other superfields to be present above the scale $M_{U}$ in order to keep the one-loop beta functions of the $\mathrm{SU}(4) \otimes \mathrm{SU}(2)_{L}$ $\otimes \mathrm{SU}(2)_{R}$ gauge group equal above this scale, and so allow string gauge unification at $M_{P}$ [15]. Since such additional superfields do not couple to the quark and lepton superfields their presence will have no effect on the LFV predictions at the one-loop level, apart from the indirect effect via the gauge couplings.

At $M_{P}$ boundary conditions were chosen to reduce the most number of independent parameters: $M_{i}=M_{1 / 2}$ (common gaugino masses), $\tilde{m}_{i}^{2}=m_{H_{u, d}}^{2}=m_{0}^{2}$ (universal soft masses), $\widetilde{\lambda_{i}}=A \lambda_{i}$ (proportional soft Yukawa matrices). With this choice we kept sources of LFV (mass splittings and CKM entries) at a minimum. A departure from the latter two conditions would introduce right from the start LFV signals therefore the results can be interpreted as the irreducible minimum amount of LFV arising from this model.

The next obvious step was to run all the above parameters again down to $M_{\text {SusY }}$, dropping terms from the RGEs involving right-handed neutrinos and sneutrinos below $M_{\nu}$. At the low energy SUSY scale we were finally able to set the superpotential Higgs parameter $\mu^{2}$ and soft Higgs boson mass $\tilde{\mu}^{2}$. These were the last parameters to be defined because they obey the following two conditions:

$$
\begin{gathered}
\mu^{2}=\frac{m_{H_{d}}^{2}-m_{H_{u}}^{2} \tan ^{2} \beta}{\tan ^{2} \beta-1}-1 / 2 m_{Z}^{2}, \\
\tilde{\mu}^{2}=1 / 2\left(m_{H_{u}}^{2}+m_{H_{d}}^{2}+2 \mu^{2}\right) \sin 2 \beta,
\end{gathered}
$$

which depend on the low-energy values of $m_{H_{u}}^{2}$ and $m_{H_{d}}^{2}$ until now unknown. The above equations simply describe how the Higgs boson VEVs are related to the (classical) renormalized Higgs potential parameters. To see how they came about we recall that after $\mathrm{SU}(2) \otimes \mathrm{U}(1)_{Y} \rightarrow U(1)_{\mathrm{QED}}$ symmetry breaking the neutral Higgs $H_{u}^{0}, H_{d}^{0}$ acquire VEVs $v_{u}, v_{d}$ therefore the Higgs potential becomes

$$
\begin{aligned}
\mathcal{V}\left(H_{u}, H_{d}\right) \rightarrow & \mathcal{V}\left(v_{u}, v_{d}\right)+(\text { physical Higgs interactions }), \\
\mathcal{V}\left(v_{u}, v_{d}\right)= & \left(\mu^{2}+m_{H_{u}}^{2}\right) v_{u}^{2}+\left(\mu^{2}+m_{H_{d}}^{2}\right) v_{d}^{2}-2 \tilde{\mu}^{2} v_{u} v_{d} \\
& +1 / 8\left(g^{\prime 2}+g^{2}\right)\left(v_{u}^{2}-v_{d}^{2}\right)^{2} .
\end{aligned}
$$

In order to recover the traditional interpretation of the VEVs, they must satisfy

$$
\frac{\partial \mathcal{V}\left(v_{u}, v_{d}\right)}{\partial v_{u}}=0, \quad \frac{\partial \mathcal{V}\left(v_{u}, v_{d}\right)}{\partial v_{d}}=0,
$$

which after simple algebraic manipulation leads to Eqs. (15), (16), except for the obvious replacement of $v_{u}, v_{d}$ by the more convenient set $\tan \beta, m_{Z}$. From Eq. (15) we see that $\mu$ is determined up to a sign, however we found that for large $\tan \beta$ this arbitrariness was not relevant.

The physical origin of LFV in this model is now clear. The Yukawa coupling matrices are effectively split into $\lambda_{u}$, $\lambda_{d}, \lambda_{e}, \lambda_{\nu}$. These matrices are not equal but related to each other by different Clebsch factors. Since $\lambda_{e} \neq \lambda_{\nu}$ one is introducing a CKM-like mixing matrix on the lepton sector which gets imprinted onto the left-handed slepton mass matrices due to the RG running between $M_{P}$ and $M_{\nu}$ (below the scale $M_{\nu}$ the terms involving the right-handed neutrinos are dropped from the RGEs). It is clear that, for example in a basis in which the charged lepton matrix $\lambda_{e}$ is diagonal the neutrino matrix $\lambda_{\nu}$ will be nondiagonal, leading to offdiagonal contributions to the left-handed slepton masses of the form

$$
\Delta \tilde{m}_{L}^{2} \sim-\frac{\ln \left(M_{P} / M_{\nu}\right)}{16 \pi^{2}} m_{0}^{2}\left(\lambda_{\nu}^{\dagger} \lambda_{\nu}\right)+\cdots
$$

It is interesting to note that in SU(5) LFV develops differently. In this model, there is no right-handed neutrino, however, LFV does arise from the presence of Higgs color triplets which mediate tree level lepto-quark interactions, which again leads to off-diagonal slepton masses due to RG running between $M_{P}$ and $M_{U}$. $\mathrm{SO}(10)$ is an example in which both the mentioned LFV processes are active $[2,11]$.

\section{THE PROCESSES $\mu \rightarrow e+\gamma$ AND $\tau \rightarrow \mu+\gamma$}

\section{A. Formalism}

The effective Lagrangian and branch ratio for the decay $\mu \rightarrow e+\gamma$ are given by

$$
\begin{gathered}
\mathcal{L}=1 / 2 \bar{u}_{e}(p-q)\left\{A_{R} P_{R}+A_{L} P_{L}\right\} \sigma^{\alpha \beta} u_{\mu}(p) \mathcal{F}_{\alpha \beta}, \\
\operatorname{BR}(\mu \rightarrow e+\gamma)=\frac{12 \pi^{2}}{G_{F}^{2} m_{\mu}^{2}}\left(\left|A_{R}\right|^{2}+\left|A_{L}\right|^{2}\right) .
\end{gathered}
$$

In their most general form, the one-loop amplitudes $A_{R}$ $=\Sigma A_{R_{i}}, A_{L}=\Sigma A_{L_{i}}$ are given by a sum of many terms [6] 
most of which of negligible importance. For the sake of simplicity we consider only the dominant contributions:

$$
\begin{aligned}
A_{R_{1}}= & \frac{e}{16 \pi^{2}} \frac{x}{\sqrt{2}}\left(\widetilde{U}_{L L}^{n \dagger}\right)_{e A}\left(\widetilde{U}_{L L}^{n}\right)_{A \mu} J_{21 A}, \\
A_{R_{2}}= & -\frac{e}{16 \pi^{2}} \frac{x}{2}\left(\widetilde{U}_{L L}^{l \dagger}\right)_{e A}\left(\widetilde{U}_{L L}^{l}\right)_{A \mu}\left(H_{32 A}+H_{31 A}\right) \\
& -\frac{e}{16 \pi^{2}} \frac{x}{2}\left(\widetilde{U}_{R L}^{l \dagger}\right)_{e A}\left(\widetilde{U}_{R L}^{l}\right)_{A \mu}\left(H_{32 \tilde{A}}+H_{31 \tilde{A}}\right), \\
A_{R_{3}}= & -\frac{e}{16 \pi^{2}}\left[\left(\widetilde{U}_{L L}^{l \dagger}\right)_{e A}\left(\widetilde{U}_{L R}^{l}\right)_{A \mu} H_{11 A}\right. \\
& \left.+\left(\widetilde{U}_{R L}^{l \dagger}\right)_{e A}\left(\widetilde{U}_{R R}^{l}\right)_{A \mu} H_{11 \tilde{A}}\right], \\
A_{L_{1}}= & \frac{e}{16 \pi^{2}} x\left[\left(\widetilde{U}_{L R}^{l \dagger}\right)_{e A}\left(\widetilde{U}_{L R}^{l}\right)_{A \mu} H_{31 A}\right. \\
& \left.+\left(\widetilde{U}_{R R}^{l \dagger}\right)_{e A}\left(\widetilde{U}_{R R}^{l}\right)_{A \mu} H_{31 \tilde{A}}\right] \\
A_{L_{2}}= & -\frac{e}{16 \pi^{2}}\left[\left(\widetilde{U}_{L R}^{l \dagger}\right)_{e A}\left(\widetilde{U}_{L L}^{l}\right)_{A \mu} H_{11 A}\right. \\
& \left.+\left(\widetilde{U}_{R R}^{l \dagger}\right)_{e A}\left(\widetilde{U}_{R L}^{l}\right)_{A \mu} H_{11 \tilde{A}}\right],
\end{aligned}
$$

here written in a notation, which we now explain. In all expressions summation over the family index $A=1 \ldots 3$ is to be understood $(\widetilde{A}=A+3)$. The factor $x=m_{\mu} /\left(\cos \beta m_{W}\right)$ and the matrices $\widetilde{U}$ and the other factors occurring in these expressions are defined in Appendix C.

We now discuss the phenomenology of Eqs. (23)-(27). The amplitude $A_{L} \ll A_{R}$ because in the 422 model LFVs associated with $\tilde{m}_{e^{c}}^{2}$ are negligible compared with the ones due
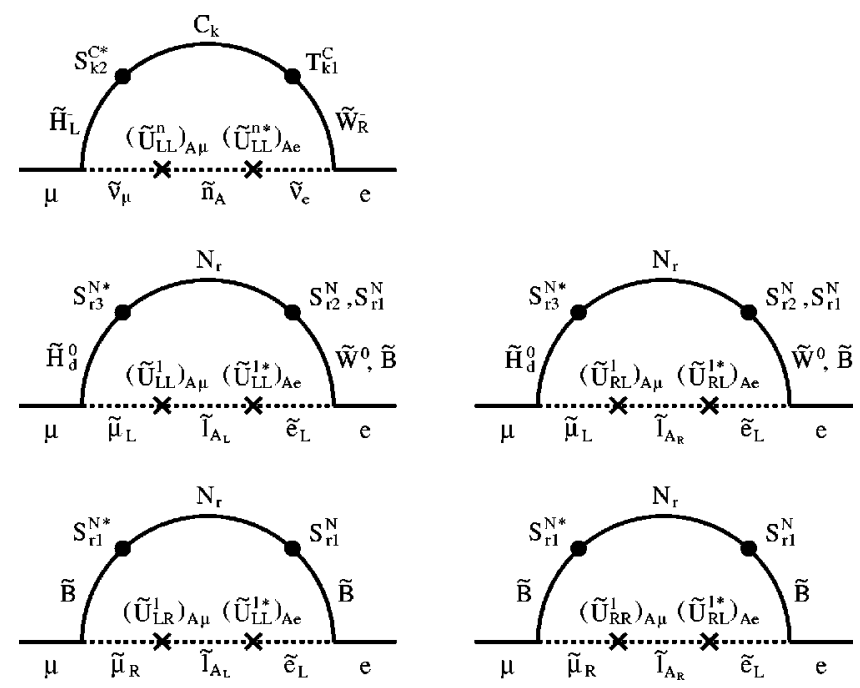

FIG. 2. Dominant supersymmetric diagrams involved in the $\mu$ $\rightarrow e+\gamma$ decay. The first, second and third row correspond to the amplitudes $A_{R_{1}}, A_{R_{2}}$ and $A_{R_{3}}$ given by Eqs. (23)-(25) respectively.

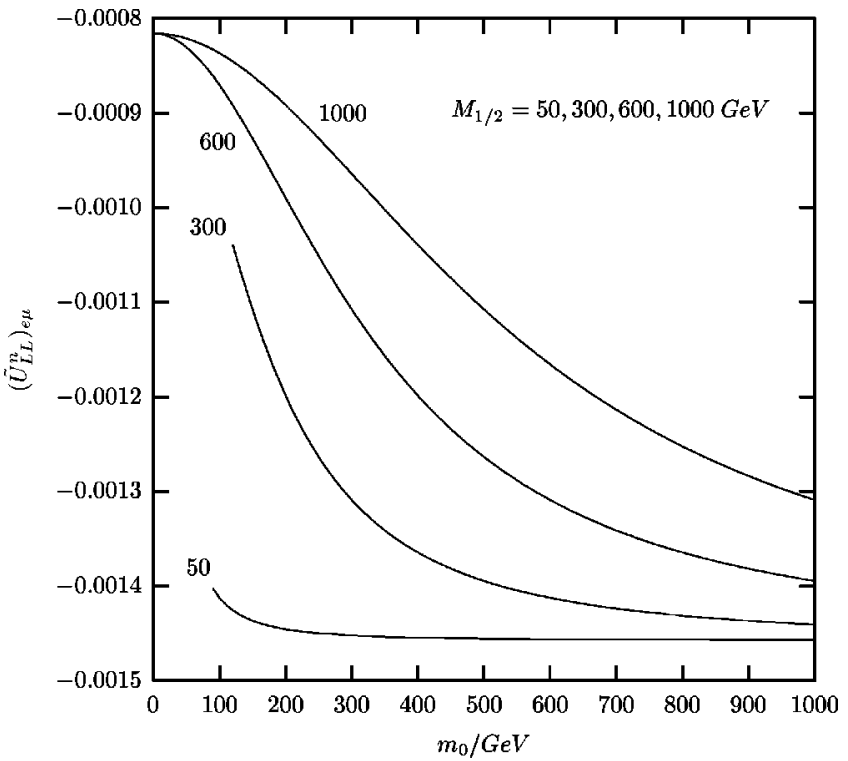

FIG. 3. Dependence of $\left(\widetilde{U}_{L L}^{n}\right)_{e \mu}$ with the universal Planck scalar mass $m_{0}$ for several values of the gaugino $\left(M_{1 / 2}\right)$ mass.

to $\tilde{m}_{L}^{2}$. Thus we will keep our attention on $A_{R_{1}}, A_{R_{2}}$ and $A_{R_{3}}$ which are associated with the diagrams in Fig. 2.

In these, a cross over slepton (dotted) line is introduced to remind us of a $\widetilde{U} \widetilde{U}$ product dependence. Similarly, the blob over the chargino- $A_{R_{1}}$ (neutralino- $A_{R_{2,3}}$ ) line stands for $S^{C} T^{C}$ $\left(S^{N} S^{N}\right)$ dependence. As an example, the explicit behavior for the dominant LFV angle is shown in Fig. 3. We stress that, though it is tempting to make a direct analogy with the perturbative mass insertion method valid when $\tan \beta$ is small,

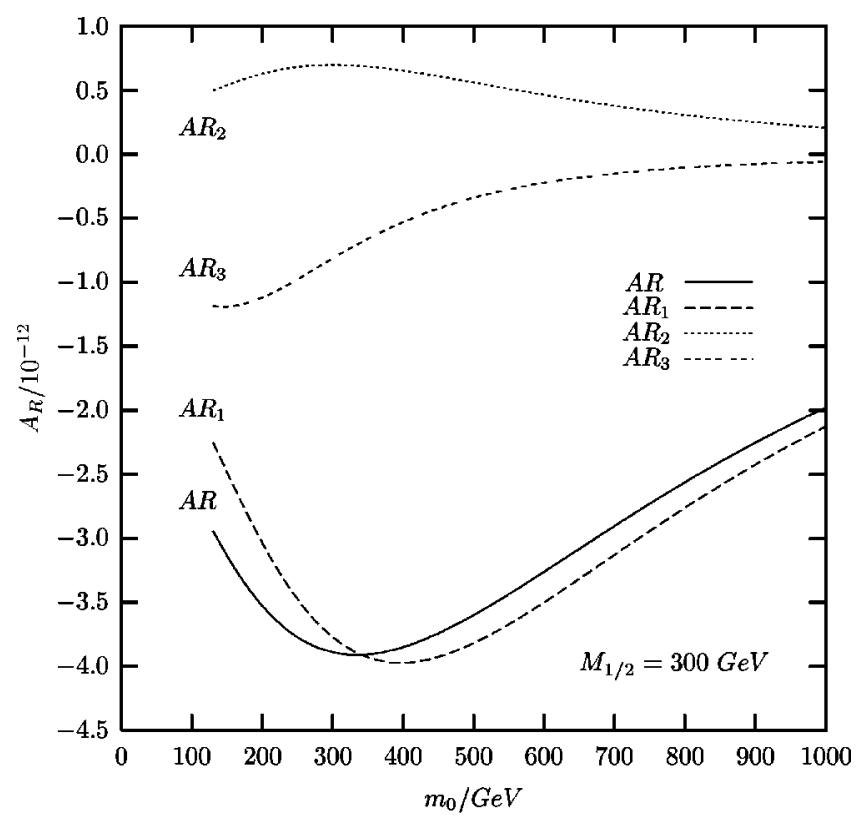

FIG. 4. Dependence of dominant amplitudes $A_{R_{i}}$ with $m_{0}$. The chargino contribution $A_{R_{1}}$ is substantially bigger than the neutralino because $A_{R_{2}}$ and $A_{R_{3}}$ are independently small and they also appear to cancel. 


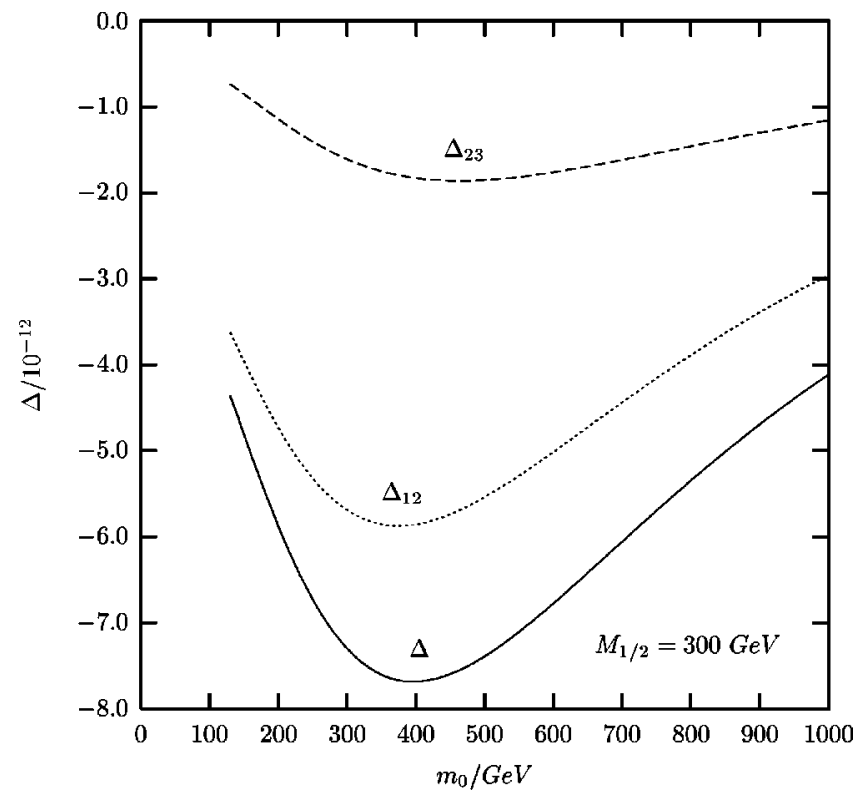

FIG. 5. Lepton flavor violation is mainly driven by the splitting of masses between the first two families as given by $\Delta_{12}$.

such comparison must be taken with great care. In models with large $\tan \beta$ and modest soft mass terms, the light supersymmetric states $\tilde{\tau}_{R}, \tilde{\tau}_{L}$ mix efficiently through $\left(\tilde{M}_{L R}^{l 2}\right)_{\tau \tau}$, $\left(\widetilde{M}_{R L}^{l 2}\right)_{\tau \tau}$ enhanced entries. Even if LFV is initially contained in the left-left charged slepton sector $\widetilde{M}_{L L}^{l 2}$, diagonalization of the full $6 \times 6$ mass matrix $\widetilde{M}^{l 2}$ forces $\widetilde{U}_{L R}^{l}, \widetilde{U}_{L R}^{l}$ and $\widetilde{U}_{R R}^{l}$ to develop non-negligible LFV signals. Figure 2 should therefore not be taken as an accurate description of the complicated physics involved but as a symbolic way of identifying the supersymmetric states that are present in each contribution to $A_{R}$.

We start by considering the neutralino amplitudes $A_{R_{2}}$

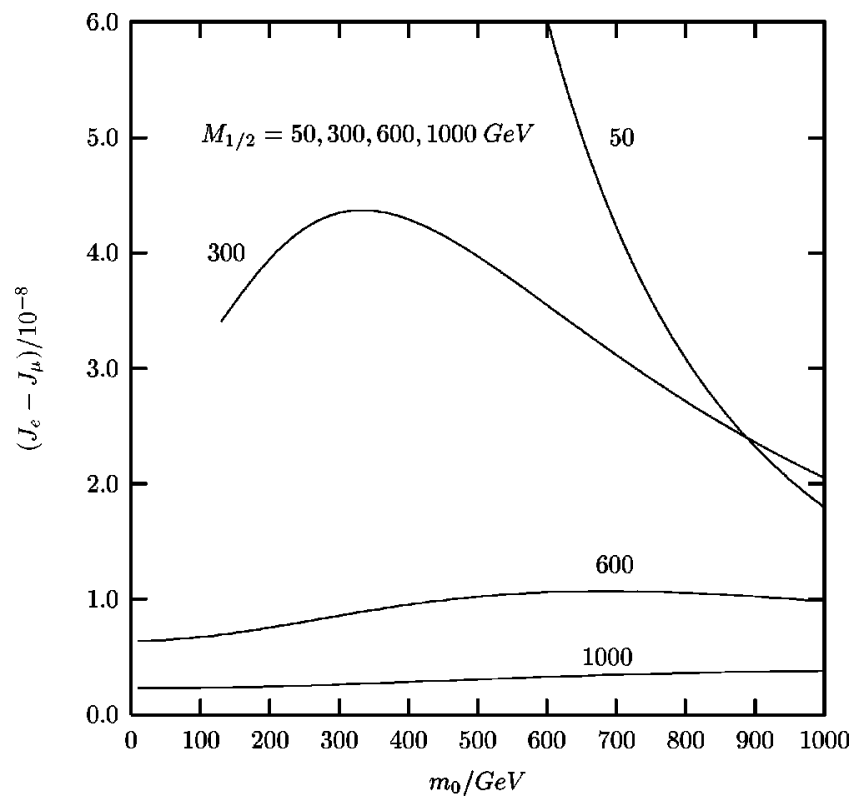

FIG. 6. Scaling of $J_{e}-J_{\mu}$ function with $m_{0}$.

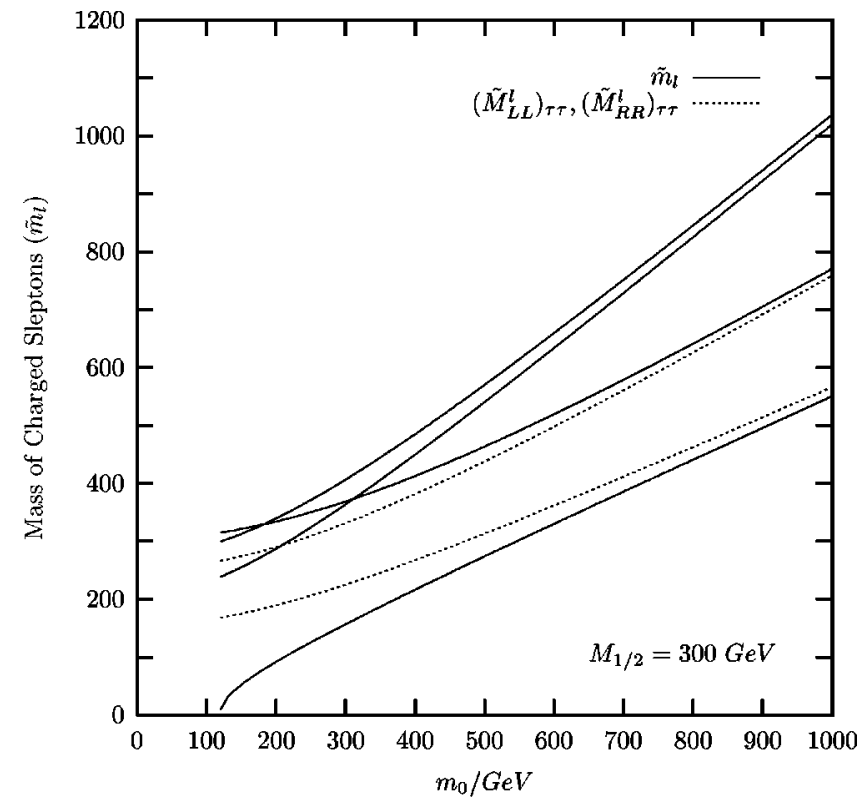

FIG. 7. Spectrum of charged sleptons $\tilde{l}$ for a range of $m_{0}$ and a selected value of gaugino mass $M_{1 / 2}$. Mixing between left and right staus is shown as a deviation of the solid line from the dotted one.

and $A_{R_{3}}$ in more detail. These are shown in Fig. 4 to be of the same order of magnitude. The amplitude $A_{R_{2}}$ accounts for LFV developed in the left-handed sector. Two sources can be identified. The first line of Eq. (24) describes LFV contained in the left-handed sector itself. The other contribution, the second line in Eq. (24), is generally significantly smaller. Its origin is associated with the fact that due to substantial stau mixing, some of the LFV originally in $\widetilde{M}_{L L}^{l 2}$ has, after diagonalization, "leaked" to the right-handed sector. This signal can only be recovered if two "chirality" flipping $\widetilde{U}_{R L}^{l}$ matrices are introduced. The amplitude $A_{R_{3}}$ accounts for LFV that is associated with mixing of charged sleptons of different "chirality." These mixings can occur either in the left-right or right-left variety corresponding to the two terms in Eq. (25).

The chargino contribution is given by $A_{R_{1}}$. Figure 4 shows that this amplitude is the most significant to $A_{R}$ and therefore to the decays $\mu \rightarrow e+\gamma$ and $\tau \rightarrow \mu+\gamma$. Its dominance over the neutralino contributions can be traced to the function $J_{21 A}$ in Eq. (23). ${ }^{6}$ When $\tan \beta$ is large $J_{21 A}$ approximately scales with $S_{12}^{C} \sim M_{12}^{C} \sim m_{W} s_{\beta}$. On the other hand, the $H_{p q A}$ functions in Eq. (24) are comparatively suppressed since they scale as $m_{W} c_{\beta}$. The amplitude $A_{R_{3}}$, while not suppressed by the diagonal $H_{11 A}$ entries, cannot compete with $A_{R_{1}}$ because of small "chirality" flipping $\widetilde{U}_{L R}^{l}, \widetilde{U}_{R L}^{l}$ matrices.

Taking the above comments in mind, we analyze in more detail the behavior of $A_{R_{1}}$ itself. Defining $\Delta=$ const $\times A_{R_{1}}$ $=\Delta_{12}+\Delta_{23}\left(J_{A}=J_{21 A}\right.$ and using approximate unitarity of $\widetilde{U}_{L L}^{n}$ ) gives

${ }^{6}$ Defined in Appendix C by Eq. (C18). 


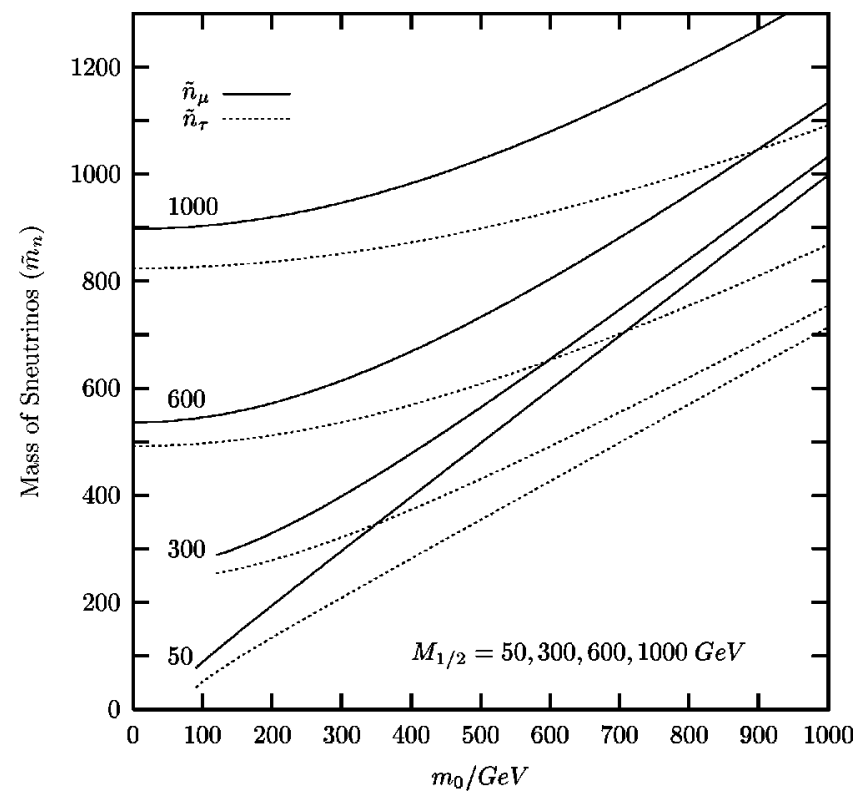

FIG. 8. Spectrum of sneutrinos $\tilde{n}_{\mu}$ and $\tilde{n}_{\tau}$ as a function of $m_{0}$ for several values of $M_{1 / 2}$.

$$
\begin{aligned}
& \Delta_{12}=-\left(\tilde{U}_{L L}^{n \dagger}\right)_{e e}\left(\tilde{U}_{L L}^{n}\right)_{e \mu}\left(J_{e}-J_{\mu}\right), \\
& \Delta_{23}=-\left(\tilde{U}_{L L}^{n \dagger}\right)_{e \tau}\left(\tilde{U}_{L L}^{n}\right)_{\tau \mu}\left(J_{\mu}-J_{\tau}\right) .
\end{aligned}
$$

The above results allow a direct interpretation of LFV in terms of $e-\mu, \mu-\tau$ splittings in Fig. 5 and $J_{e}-J_{\mu}$ whose scaling with $m_{0}$ is shown in Fig. 6. If $\tan \beta$ is small then $\left|\Delta_{23}\right| \gg\left|\Delta_{12}\right|$, while as Fig. 5 shows for large $\tan \beta$ over much of parameter space we find $\left|\Delta_{12}\right| \gg\left|\Delta_{23}\right|$. The empirical effect we observe seems to be related to the large $\tan \beta$ result that $\lambda_{\mu} \gg \lambda_{\text {charm }}$, which tells us that second family Yukawa couplings receive an overall enhancement and this is respon-

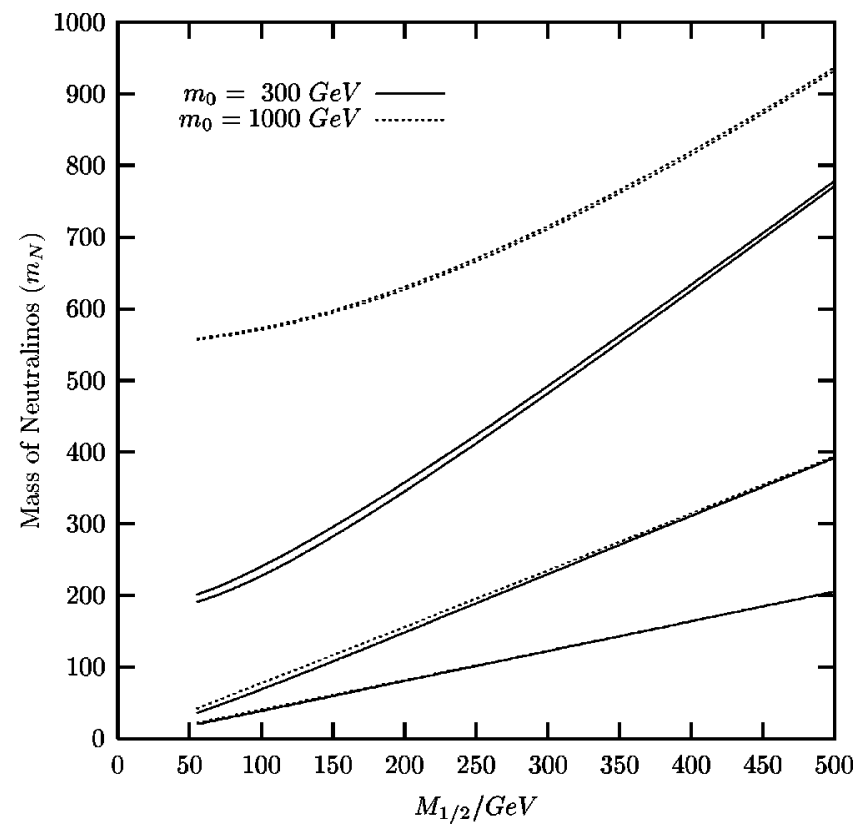

FIG. 9. Spectrum of neutralinos as a function of $M_{1 / 2}$.

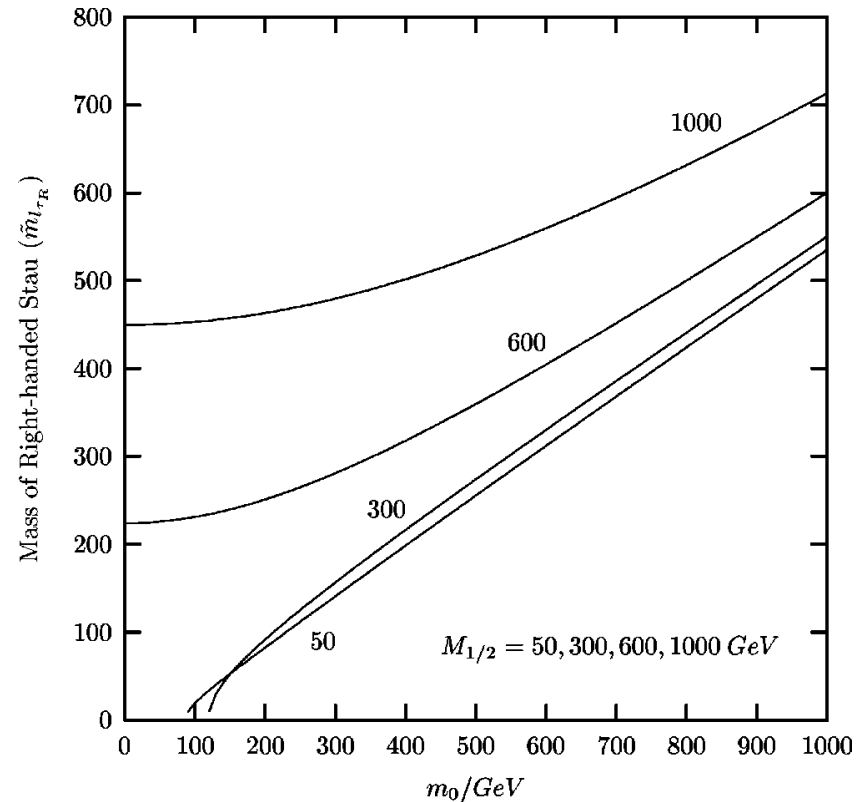

FIG. 10. Mass of lightest scalar sparticle, namely the righthanded stau $\left(\widetilde{l}_{\tau_{R}}\right)$.

sible for the increase in 1-2 family splittings. It turns out that the 2-3 family mixing is also substantially increased in this model, but for $\mu \rightarrow e+\gamma$ this effect is destroyed by the small $(1,3)$ family mixing factor. For $\tau \rightarrow \mu+\gamma$, on the other hand, the rate here is controlled exclusively by $(2,3)$ family mixing and we find a large enhancement in this case.

\section{B. Overview of results}

In our numerical results we assumed $\alpha_{s}=0.115$ and $m_{\text {bottom }}=4.25 \mathrm{GeV}$. An increase in $\alpha_{s}\left(m_{\text {bottom }}\right)$ leads to smaller slepton masses (bigger $e-\mu$ splitting) therefore to an enhancement of LFV. The parameters we made to vary were $M_{1 / 2}, m_{0}, A_{0}$ and $M_{\nu}$. When not explicitly mentioned the graphs refer to default values of $A_{0}=0 \mathrm{GeV}$ and $M_{\nu}$ $=M_{U}$.

In Fig. 7 we plotted the slepton spectrum $\tilde{m}_{l}$. Because of the large $\tan \beta$, for decreasing $m_{0}$, we verify that the lightest slepton $\widetilde{l}_{\tau_{R}}$ is rapidly driven negative, on the other hand $\widetilde{l}_{\tau_{L}}$ is pushed upwards and forced to be the heaviest sparticle. These phenomena are absent in conventional low $\tan \beta$ models. Figure 8 displays the masses of the muon and tau sneutrinos. The splitting between them ensures that $\Delta_{23}$ in Eq. (29) does not vanish. The neutralino particle spectrum is shown in Fig. $9^{7}$ and the mass of the right-handed stau in

\footnotetext{
${ }^{7}$ When $m_{0}, M_{1 / 2}>m_{Z}$, the heaviest chargino is approximately degenerate with the two heaviest neutralinos (which scale with $\mu$, the Higgs mixing parameter), while the lightest chargino has the same mass as the second lightest neutralino (both scale with $M_{2}$ ). Finally the lightest neutralino scales with $M_{1}$. These three sets are in close correspondence with the scaling of Higgsinos, $W$-inos and $B$-ino.
} 


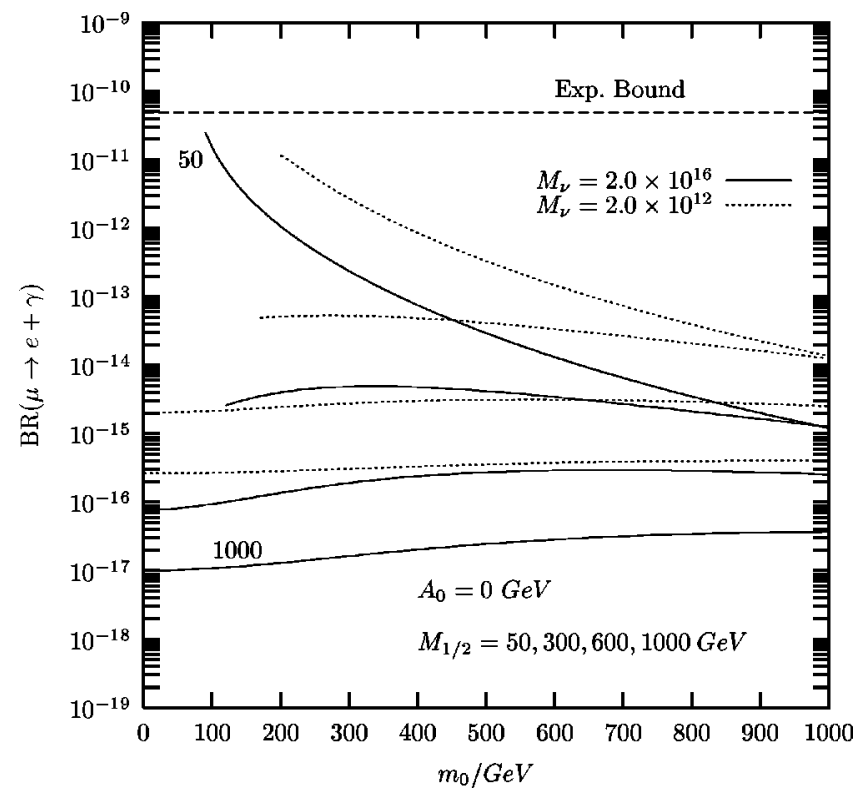

FIG. 11. Branch ratio for the decay $\mu \rightarrow e+\gamma$ as function of $m_{0}$ for several values of $M_{1 / 2}$ and two $M_{\nu}$ scales.

Fig. 10 .

We now consider the branch ratio $\mu \rightarrow e+\gamma$. In Fig. 11 we plot its dependence with $m_{0}$ for selected values of $M_{1 / 2}$ (and just two extreme values of $M_{\nu}$ ). Discontinued lines signal regions where $\tilde{m}_{l_{\tau_{R}}} \rightarrow 0$. When $M_{1 / 2}$ is not much bigger than $m_{0}$, the $\operatorname{BR}(\mu \rightarrow e+\gamma)$ increases with decreasing $m_{0}$ for in this limit the sneutrinos are getting lighter. The present experimental bound $\operatorname{BR}(\mu \rightarrow e+\gamma)<4.9 \times 10^{-11}$ is close to the most enhanced decays, however it still does not rule out any region of the $M_{1 / 2}, m_{0}$ and $M_{\nu}$ parameter space.

Figure 12 gives the branch ratio dependence on $M_{\nu}$. We

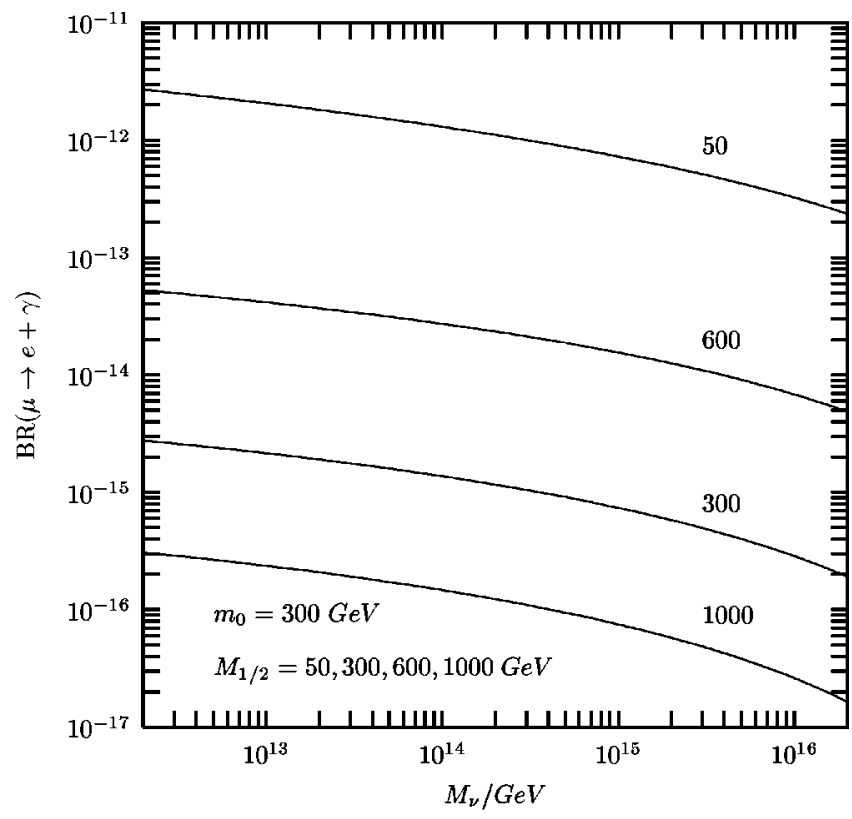

FIG. 12. Branch ratio for $\mu \rightarrow e+\gamma$ versus $M_{\nu}$.

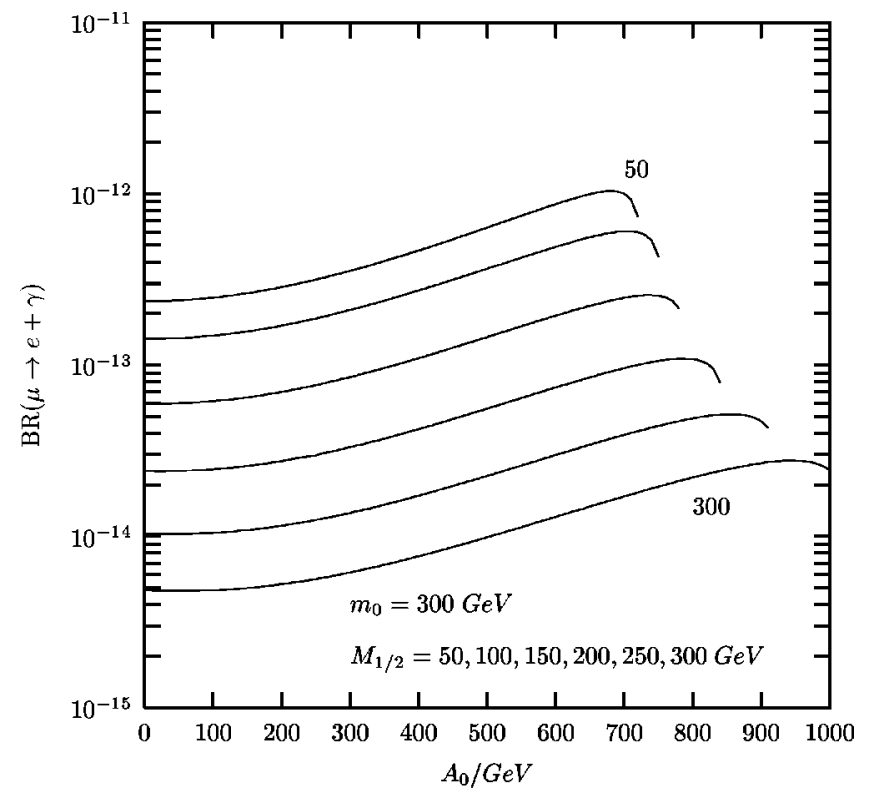

FIG. 13. Branch ratio of $\mu \rightarrow e+\gamma$ as function of $A_{0}$.

can see that the rate $\mu \rightarrow e+\gamma$ increases for decreasing $M_{\nu}$. This behavior is easily understood; models with lower $M_{\nu}$ allow right-handed neutrinos to decouple at lower energies, which in turn, forces $\tilde{m}_{L}^{2}$ to be under radiative LFV contributions for an extended $M_{P} \rightarrow M_{\nu}$ energy range as described in Eq. (20). The variations with $A_{0}$ are plotted in Fig. 13. When $\tan \beta$ is big the sleptons mass matrix is explicit independent of $\widetilde{\lambda_{e, \nu}}$. Since RGEs care only about ${\widetilde{\lambda_{e, \nu}}}_{\text {, we see }}$ that the results must be approximately invariant under $A_{0}$ $\rightarrow-A_{0}$. For that reason we have only considered $A_{0}>0$. In general, $A_{0}$ does not affect the branch ratio decay significantly. A variation of three orders of magnitude in $A_{0}$ leads only to one magnitude enhancement in $\operatorname{BR}(\mu \rightarrow e+\gamma)$.

In Fig. 14 we show the $\operatorname{BR}(\tau \rightarrow \mu+\gamma)$ which is experimentally constrained to be less than $4.2 \times 10^{-6}$. This limit already imposes some constraints in the allowed SUSY parameter space for this model, particularly when $M_{\nu}$ is allowed to take values as low as $10^{12} \mathrm{GeV}$.

\section{Results near the experimental limits}

It is clear from the results so far that the interesting region of parameter space from the point of view of the LFV processes corresponds to relatively low values of soft SUSY breaking parameters, say $m_{0}<500 \mathrm{GeV}$ and $M_{1 / 2}$ $<200 \mathrm{GeV}$. In this subsection we shall concentrate on this region, and examine the relationship between LFV processes and direct experimental bounds on the particle mass limits coming from the CERN $e^{+} e^{-}$collider LEP, for example.

In Fig. 15 we show the branch ratio for $\mu \rightarrow e+\gamma$ for a range of $m_{0}<500 \mathrm{GeV}$ and several values of $M_{1 / 2}$ $<200 \mathrm{GeV}$, taking two extreme values of right-handed neutrino mass. As the experimental bound improves it is clear how increasingly larger regions of the $m_{0}-M_{1 / 2}$ plane in this model may be excluded, with the low values of right-handed neutrino mass (well motivated from neutrino physics) pro- 


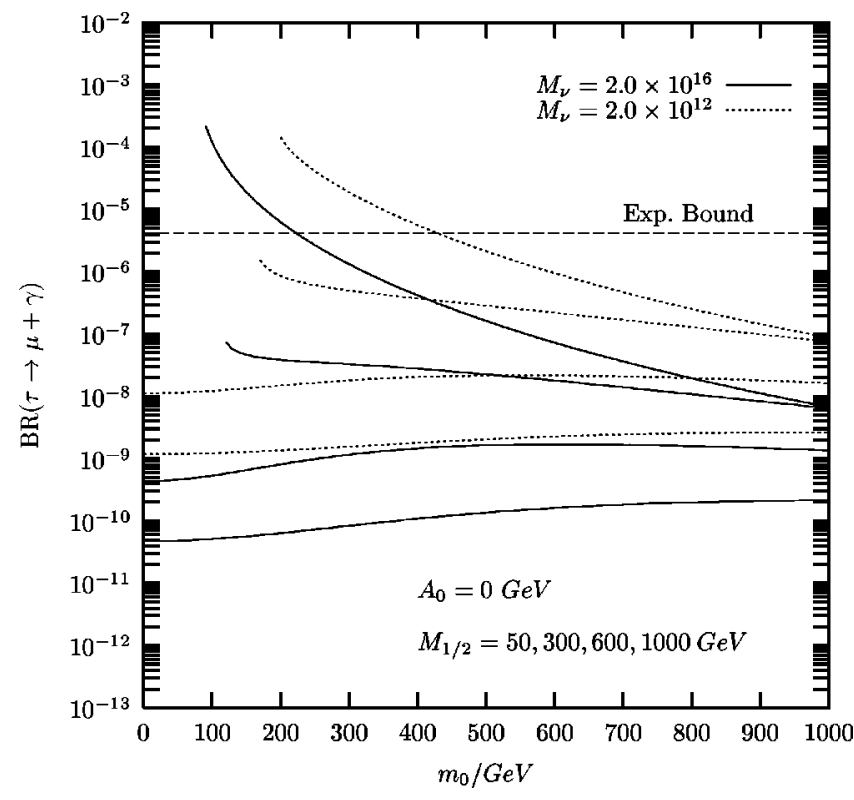

FIG. 14. Branch ratio of $\tau \rightarrow \mu+\gamma$ for a range of $m_{0}$ and several values of $M_{1 / 2}$.

viding the larger rates closer to the experimental limit.

Figure 16 shows the predicted branch ratio for $\tau \rightarrow \mu+\gamma$. The well-motivated $M_{\nu}=2 \times 10^{12} \mathrm{GeV}$ curves clearly already provide useful constraints on the SUSY particle spectrum.

Figure 17 shows the dependence of $\tau \rightarrow \mu+\gamma$ on the right-hand neutrino scale- $M_{\nu}$. One can see that, as $M_{\nu}$ decreases, the right-handed neutrino decouples at a lower energy, thus allowing additional LFV to be generated through

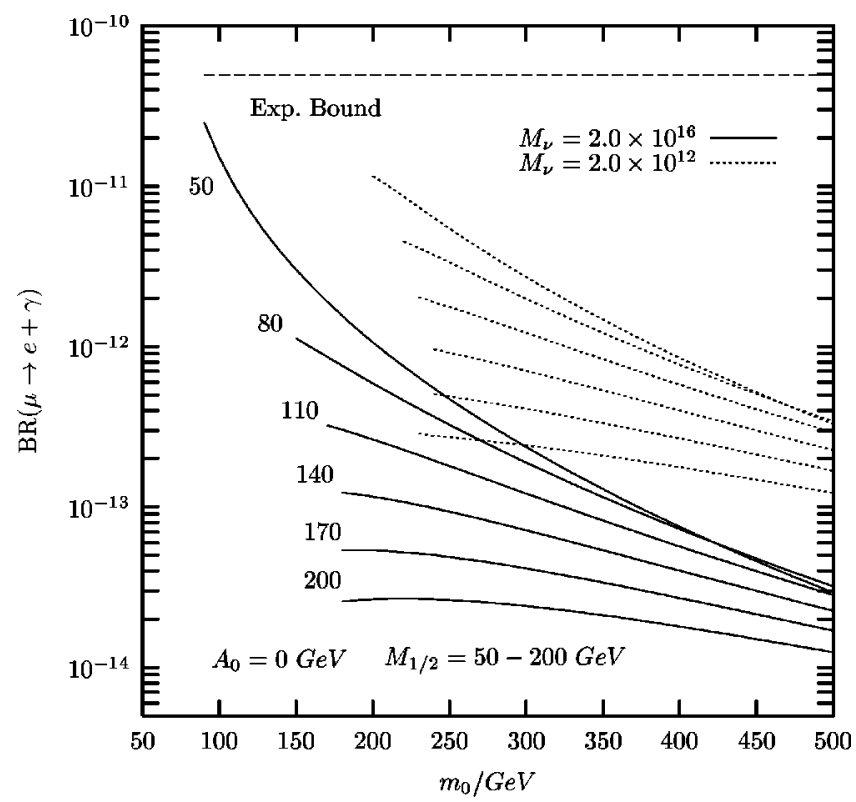

FIG. 15. Branch ratio for $\mu \rightarrow e+\gamma$ for a range of $m_{0}<500 \mathrm{GeV}$ and several values of $M_{1 / 2}<200 \mathrm{GeV}$. Two extreme values of $M_{\nu}$ are displayed: solid lines correspond to $M_{\nu}=M_{\mathrm{GUT}}$, while dotted lines to $M_{\nu}=2 \times 10^{12} \mathrm{GeV}$.

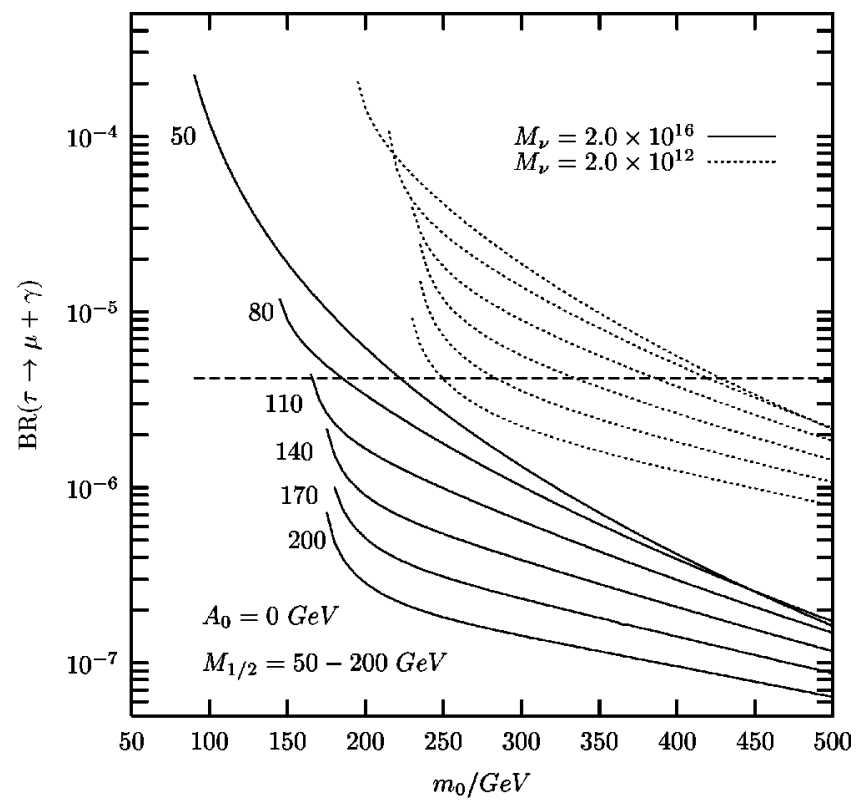

FIG. 16. Branch ratio for $\tau \rightarrow \mu+\gamma$ for a range of $m_{0}$ $<500 \mathrm{GeV}$ and several values of $M_{1 / 2}<200 \mathrm{GeV}$. Two extreme values of $M_{\nu}$ are displayed: solid lines correspond to $M_{\nu}=M_{\mathrm{GUT}}$, while dotted lines to $M_{\nu}=2 \times 10^{12} \mathrm{GeV}$.

the RGE running, which leads to an enhancement in the branch ratio.

In this model the spectrum is completely determined by the values of the input parameters, in particular $m_{0}$ and $M_{1 / 2}$, with very little sensitivity to $M_{\nu}$, for example. It is clearly of interest to compare the direct experimental limits which may be placed in these parameters, from the CERN $e^{+} e^{-}$collider LEP for example, to the indirect limits coming

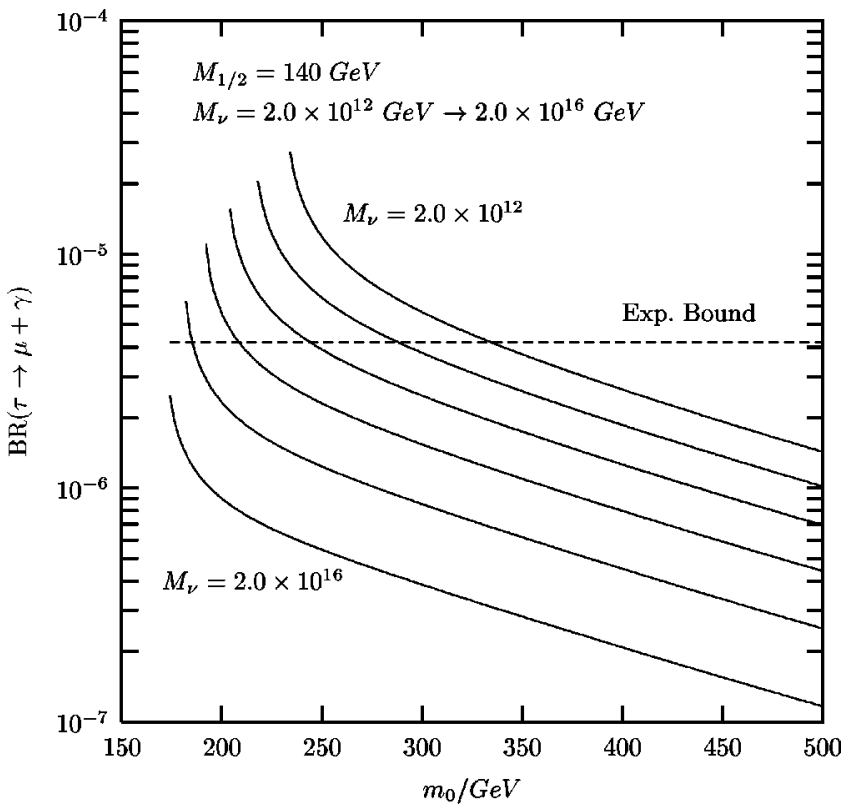

FIG. 17. Branch ratio for $\tau \rightarrow \mu+\gamma$ for a range of $m_{0}$ $<500 \mathrm{GeV}$ and $M_{1 / 2}=140 \mathrm{GeV}$. The six curves plotted correspond to equally log-scaled intervals of $M_{\nu}$ in the range $2 \times 10^{12}$ to $2 \times 10^{16} \mathrm{GeV}$. 


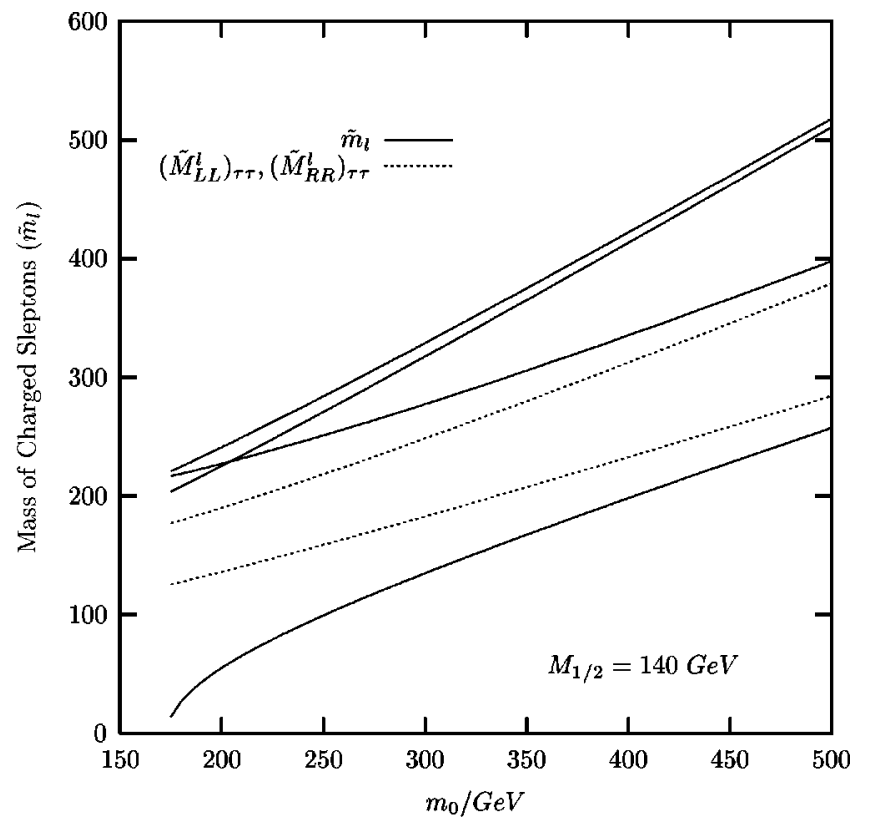

FIG. 18. Spectrum of charged sleptons $(\widetilde{l})$ for a range of $m_{0}$ and a fixed low value of $M_{1 / 2}=140 \mathrm{GeV}$. Mixing between left and right staus is shown as a deviation of the solid from the dotted one $\left(A_{0}=0 \mathrm{GeV}, M_{\nu}=2.0 \times 10^{16} \mathrm{GeV}\right)$.

from the LFV processes we have considered. Therefore we present a series of plots which give a detailed exposition of the sparticle spectrum in the low mass region where experiments are sensitive to LFV processes.

We begin in Fig. 18 by showing the spectrum of charged sleptons for a fixed low value of $M_{1 / 2}=140 \mathrm{GeV}$ corresponding to charginos in the unexplored LEP2 range 95-105 $\mathrm{GeV}$ (as we shall see shortly). The plot shows that the lightest charged slepton mass ranges from 125-260 GeV over the region of $m_{0}=280-500 \mathrm{GeV}$ allowed in a scenario in which the LFV bound for the $\tau$ decay has improved to $4.2 \times 10^{-7} \mathrm{GeV}$. Figure 19 shows the very weak dependence of the lightest slepton mass on $M_{1 / 2}$. The corresponding sneutrino masses in Fig. 20 have a similar mass dependence but are somewhat heavier.

The strongest constraint on $M_{1 / 2}$ comes from the lightest charginos and neutralinos in Fig. 21. The full spectrum of charginos and neutralinos, for a fixed value of $m_{0}$ $=300 \mathrm{GeV}$, and varying $M_{1 / 2}$, is shown in Fig. 22 .

The current published LEP2 limit on chargino masses is around $85 \mathrm{GeV}$ [24-27]. This bound does not include analysis of the most recent runs, which will increase this limit to about $94 \mathrm{GeV}$ [28]. A chargino mass limit of $94 \mathrm{GeV}$ would correspond to $M_{1 / 2}>125 \mathrm{GeV}$ for $m_{0}$ in the intermediate 200-500 GeV range. The experimental limit on $\tau \rightarrow \mu+\gamma$ would need to be increased by one order of magnitude in order to be competitive with these direct limits.

Finally we present Figs. 23 and 24 that neatly summarize some of the most important results obtained. The regions bounded by the thick broken line are forbidden since they correspond to negative right-handed stau masses or to a combination of $m_{0}-M_{1 / 2}$ that renders consistent electro-weak symmetry breaking in this model impossible, i.e., $\mu^{2}$ of Eq.

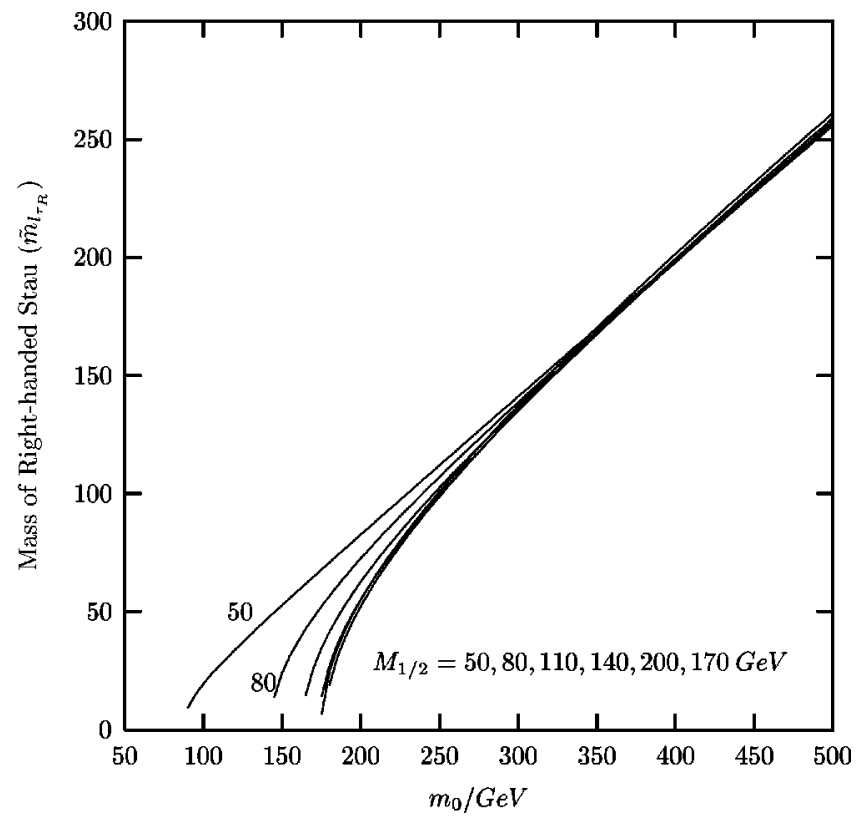

FIG. 19. Mass of lightest scalar sparticle, namely the righthanded stau $\left(\tilde{l}_{\tau_{R}}\right)$, for a range of $m_{0}<500 \mathrm{GeV}$ and several values of $M_{1 / 2}$ (note that the ordering of the $M_{1 / 2}$ lines which are plotted, from left to right, are in correspondence with the order shown on the label in the graph).

(15) negative. The regions below the dotted lines labeled by $4.2 \times 10^{-6}\left(4.2 \times 10^{-7}\right)$ are excluded by the current (one order of magnitude improved) bound on the branch ratio of $\tau$ $\rightarrow \mu+\gamma$. The horizontal solid lines indicate the mass of the lightest neutralino, while the curved lines that extend from the "excluded region" correspond to the mass of the lightest scalar sparticle, the right-handed stau. The mapping of the

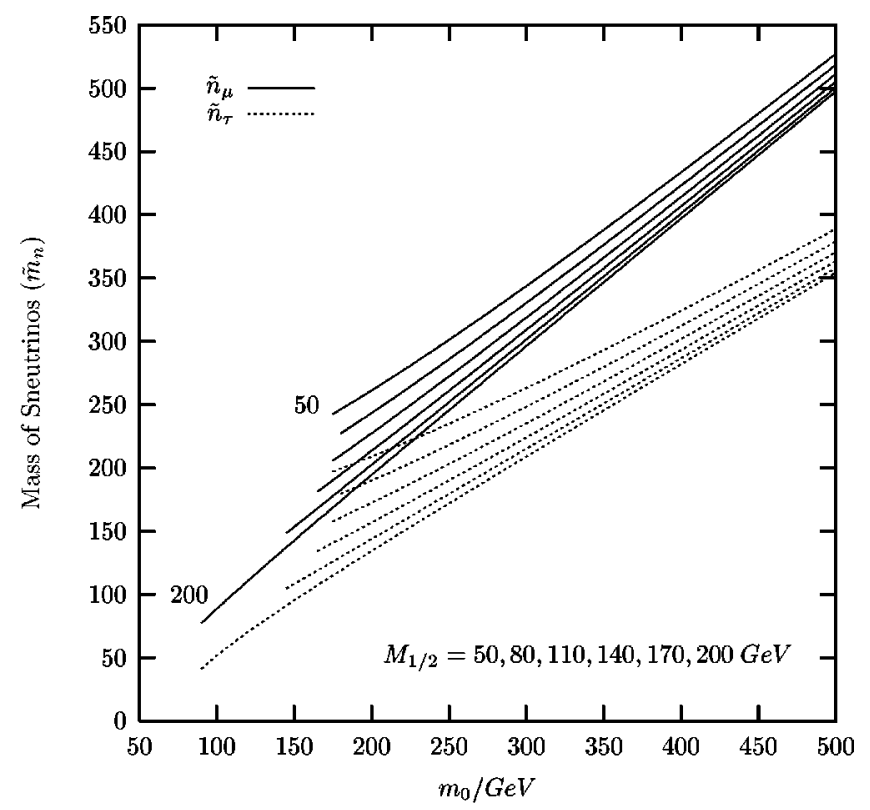

FIG. 20. Spectrum of sneutrinos $\tilde{n}_{\mu}$ and $\tilde{n}_{\tau}$ for a range of $m_{0}<500 \mathrm{GeV}$ and several values of $M_{1 / 2}\left(A_{0}=0 \mathrm{GeV}, M_{\nu}\right.$ $\left.=2.0 \times 10^{16} \mathrm{GeV}\right)$. 


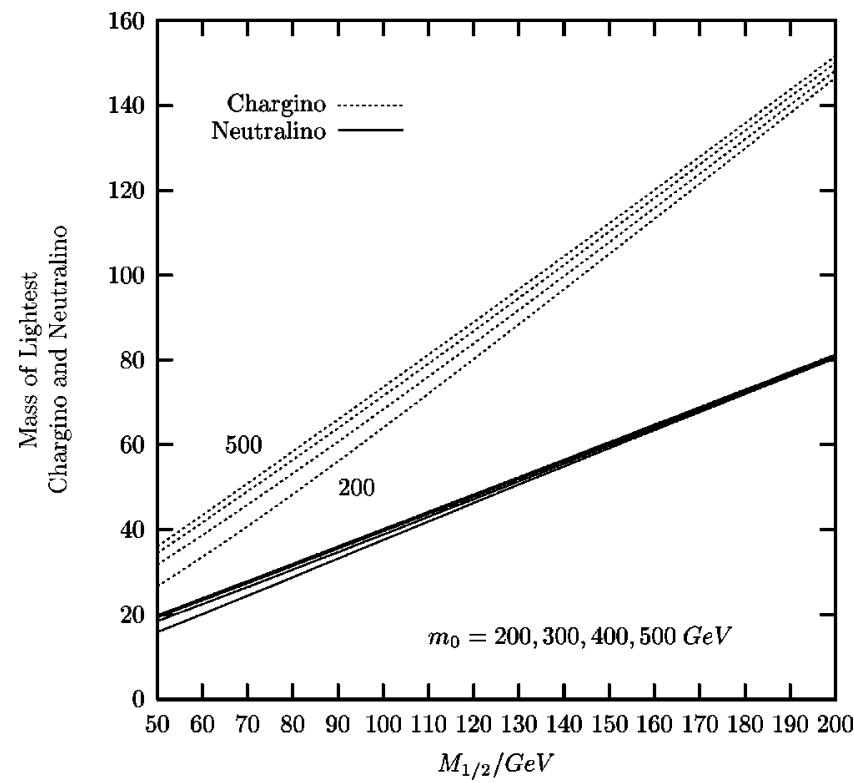

FIG. 21. Spectrum of lightest chargino and neutralino for a range of $M_{1 / 2}$ and selected values of $m_{0}<500 \mathrm{GeV}\left(A_{0}\right.$ $\left.=0 \mathrm{GeV}, M_{\nu}=2.0 \times 10^{16} \mathrm{GeV}\right)$.

$m_{0}-M_{1 / 2}$ plane into the neutralino-stau space enables the unequivocal identification of the LSP; inside (outside) the semi-circle plotted with a thin dashed line the stau (neutralino) is the LSP.

Analyzing in more detail Fig. 23, which corresponds to $M_{\nu}=2.0 \times 10^{16} \mathrm{GeV}$, we observe that the experimental bound on the branch ratio for the decay $\tau \rightarrow \mu+\gamma$ is of little practical significance. Indeed, even when this limit is improved by one order of magnitude the corresponding excluded region on the $m_{0}-M_{1 / 2}$ plane is still almost all

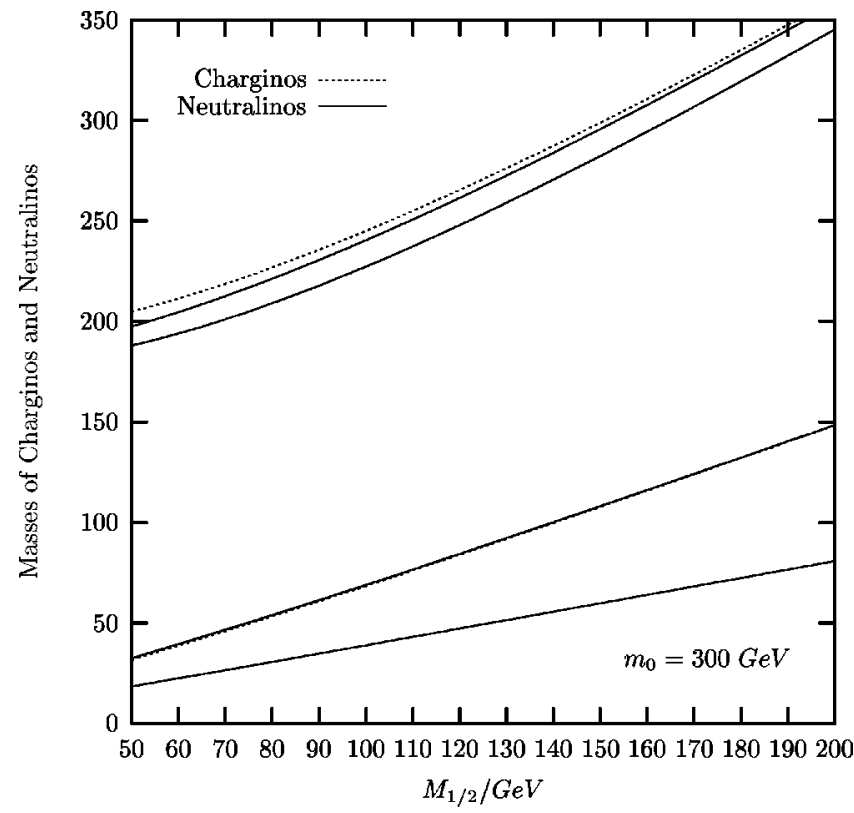

FIG. 22. Spectrum of charginos $\left(m_{C}\right)$ and neutralinos $\left(m_{N}\right)$ for a range of $M_{1 / 2}$ and $m_{0}=300 \mathrm{GeV}\left(A_{0}=0 \mathrm{GeV}, M_{\nu}=2.0\right.$ $\left.\times 10^{16} \mathrm{GeV}\right)$.

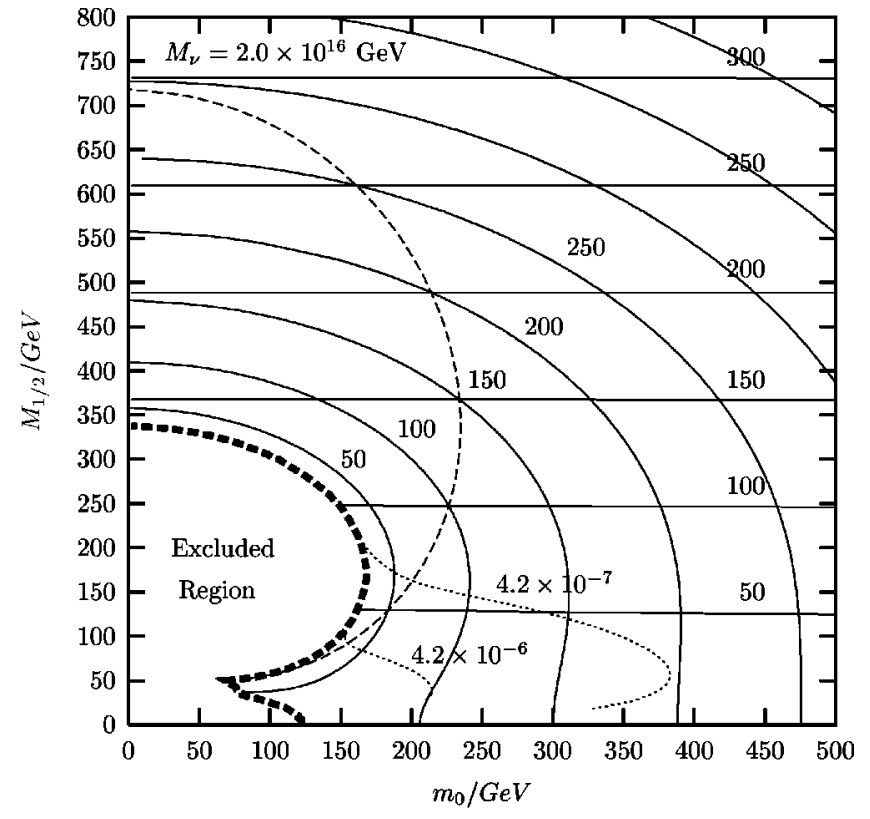

FIG. 23. Constrained $m_{0}-M_{1 / 2}$ plane when $M_{\nu}$ $=2.0 \times 10^{16} \mathrm{GeV}$. The horizontal solid lines represent lightest neutralino mass contours, and the remaining solid curves represent the right-handed stau mass contours. The region bounded by the thick broken line is excluded as it corresponds to unacceptable negative right-handed stau mass. The region below the dotted curve, labeled $4.2 \times 10^{-6}$, is excluded by the current experimental bound on the branch ratio of $\tau \rightarrow \mu+\gamma$. The LEP2 bound on the chargino mass $>94 \mathrm{GeV}$ excludes $M_{1 / 2}<125 \mathrm{GeV}$. The lower bound on the stau mass is $72 \mathrm{GeV}[29,30]$.

within the band $M_{1 / 2}<125 \mathrm{GeV}$ which has, in any case, already been excluded by the present lower bound on the chargino mass. However, from Fig. 24, obtained when $M_{\nu}$ $=2.0 \times 10^{12} \mathrm{GeV}$, we can clearly see that as the bound on

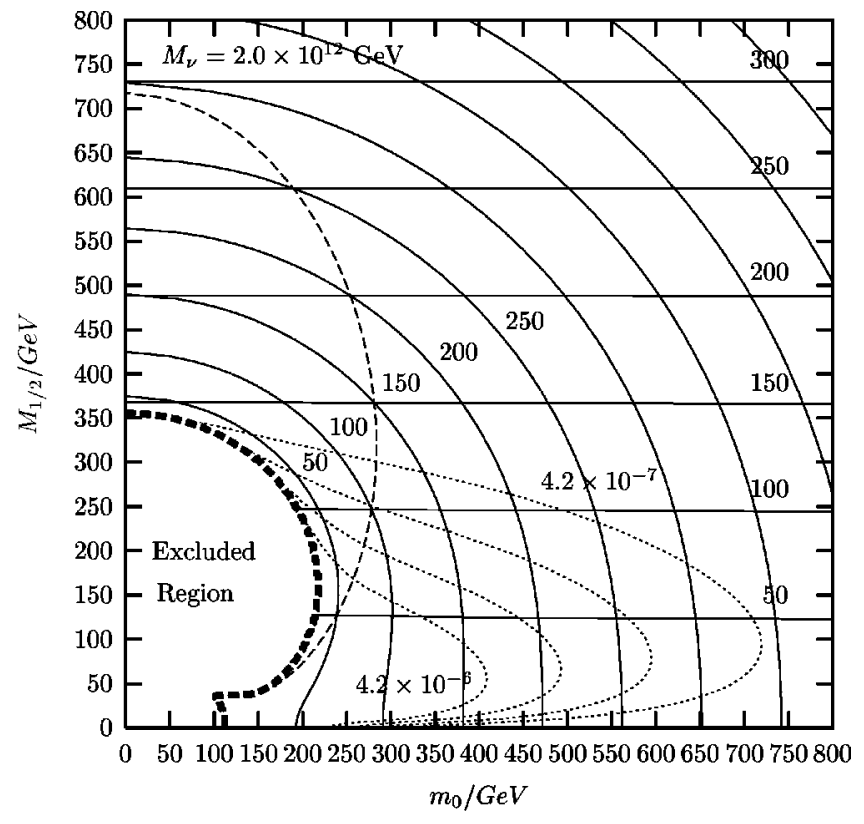

FIG. 24. Constrained $m_{0}-M_{1 / 2}$ plane when $M_{\nu}$ $=2.0 \times 10^{12} \mathrm{GeV}$. The solid and dashed contours are as described in Fig. 23. 
$\tau \rightarrow \mu+\gamma$ decreases, the regions with lowest $m_{0}, M_{1 / 2}$ are increasingly excluded beyond the $M_{1 / 2}=125 \mathrm{GeV}$ frontier.

\section{CONCLUSIONS}

The main qualitative conclusion of this study is that LFV is not a unique prediction of SUSY GUTs, but is also found in certain string-inspired models which do not possess a simple gauge group. In order to illustrate this we have calculated the minimum irreducible contributions to LFV in the string-inspired minimal $\mathrm{SU}(4) \otimes \mathrm{SU}(2)_{L} \otimes \mathrm{SU}(2)_{R}$ model. The main features of this model are large $\tan \beta$ and neutrino masses with an intermediate mass scale $M_{\nu}$. The mechanism responsible for LFV in the 422 model is similar to the one in $\mathrm{MSSM}+\nu$ but here involves a much more constrained parameter space, leading to a range of $\tan \beta$ outside that previously considered. Also previous studies on the fermion mass spectrum in this model lead to a set of well defined mixing angles which enable precise predictions of LFV to be made. The dominant contribution was seen to come from the amplitude $A_{R_{1}}$ corresponding to sneutrinos and charginos in the loop, with the LFV controlled by the off-diagonal contributions to the left-handed sneutrino mass squared matrix. The LFV in this model originates from off-diagonal contributions to the soft sneutrino mass matrix which are generated by RGE evolution in the high energy region between the Planck and the right-handed neutrino scale.

The main quantitative conclusion is that the LFV rates in these models are substantially enhanced compared to other models. This conclusion is based on values of mixing angles taken from previous studies of the fermion mass spectrum in this model. The enhancement effect is well illustrated by the detailed analysis of the parameter space near the current experimental limits given in Figs. 15-24. In particular we find that the current limit on $\tau \rightarrow \mu+\gamma$ is very close to the predictions of this model, especially for the lower values of right-handed neutrino masses which are well motivated by the physics of neutrino masses. If the experimental bounds on $\tau \rightarrow \mu+\gamma$ were improved by one order of magnitude then this model would become severely constrained, providing a decisive test of such models. Since we have concentrated on the minimum irreducible amount of LFV in the model, failure to observe $\tau \rightarrow \mu+\gamma$ at its predicted rate would enable such models to be experimentally excluded. More optimistically a direct observation of $\tau \rightarrow \mu+\gamma$ could provide an indirect discovery of supersymmetry in general and large $\tan \beta$ string-inspired models in particular.

\section{ACKNOWLEDGMENTS}

The work of M.O. was supported by JNICT under contract grant: PRAXIS XXI/BD/5536/95. The authors would like to thank M. E. Gómez for calling our attention to an error in a previous version of the paper, concerning the light left-handed sneutrino mass matrix, and indicating its correction as in Ref. [31].

\section{APPENDIX A: EFFECTIVE THEORY BELOW $M_{P}$}

Below the 422 breaking scale $M_{U} \sim 10^{16} \mathrm{GeV}$, the model effectively reduces to the MSSM $+\nu$ model. The effective
Lagrangian is given by summing the superpotential, scalar potential, scalar and gaugino mass contributions $\mathcal{L}=\mathcal{L}_{W}-\mathcal{V}$ $-\mathcal{L}_{m}-\mathcal{L}_{\lambda}$, each of which we have written in the following form:

$$
\begin{aligned}
& \mathcal{W}=u^{c} \lambda_{u} Q H_{u}+d^{c} \lambda_{d} Q H_{d}+\nu^{c} \lambda_{\nu} L H_{u}+e^{c} \lambda_{e} L H_{d}+\mu H_{u} H_{d} \\
& +1 / 2 M_{\nu} \nu^{c} \nu^{c} \\
& \mathcal{V}=\widetilde{u}^{c} \widetilde{\lambda_{u}} \widetilde{Q} H_{u}+\widetilde{d}^{c} \tilde{\lambda_{d}} \widetilde{Q} H_{d}+\widetilde{\nu}^{c} \widetilde{\lambda_{\nu}} \widetilde{L} H_{u}+\widetilde{e}^{c} \tilde{\lambda_{e}} \widetilde{L} H_{d} \\
& +\tilde{\mu}^{2} H_{u} H_{d}+\text { H.c., } \\
& +1 / 2 \widetilde{\nu}^{c} \tilde{m}_{\nu^{c}}^{2} \widetilde{\nu}^{c *}+1 / 2 \widetilde{e}^{c} \tilde{m}_{e^{c}}^{2} \widetilde{e}^{c *}+1 / 2 \tilde{M}_{\nu}^{2} \widetilde{\nu}^{c} \widetilde{\nu}^{c}+\text { H.c., }
\end{aligned}
$$

$\mathcal{L}_{\lambda}=1 / 2 M_{1} \overline{\widetilde{B}} \widetilde{B}+1 / 2 M_{2} \overline{\widetilde{W}}_{a} \widetilde{W}_{a}+1 / 2 M_{3} \overline{\widetilde{G}}_{x} \widetilde{G}_{x}$,

which defines our conventions and notation for the soft parameters in the low energy effective theory.

\section{APPENDIX B: RGES}

This appendix lists the one loop RGEs which we used to run the parameters between $M_{U}$ and $M_{P}$ using the effective 422 model as described in Sec. III. We have neglected the wave function renormalization of the GUT Higgs fields, therefore the equations resemble those of the MSSM $+\nu$ with effective Yukawa couplings $\lambda_{u}, \lambda_{d}, \lambda_{\nu}, \lambda_{e}$, but with the $\mathrm{SU}(4) \otimes \mathrm{SU}(2)_{L} \otimes \mathrm{SU}(2)_{R}$ gauge group instead of the standard model gauge group.

The gauge group factors are given by

\begin{tabular}{lrrrrrrr} 
Group & $i$ & $b_{i}$ & $c_{i}^{\lambda}$ & $c_{i}^{h}$ & $c_{i}^{2 h}$ & $c_{i}^{F}$ & $c_{i}^{F^{c}}$ \\
\hline $\mathrm{SU}(4)$ & 1 & -6 & $15 / 4$ & 0 & 0 & $15 / 8$ & $15 / 8$ \\
$\mathrm{SU}(2)_{L}$ & 2 & 1 & $3 / 2$ & $3 / 4$ & $3 / 2$ & $3 / 4$ & 0 \\
$\mathrm{SU}(2)_{R}$ & 3 & 1 & $3 / 2$ & $3 / 4$ & $3 / 2$ & 0 & $3 / 4$
\end{tabular}

The $b$ 's displayed above account only for the contributions coming from the $F, F^{c}$ and $h$ multiplets. More generally one can write

$$
\begin{aligned}
\left(b_{4}, b_{L}, b_{R}\right)= & (-6,1,1)+2 n_{H_{L}}(1,2,0)+2 n_{H_{R}}(1,0,2) \\
& +n_{D}(1,0,0),
\end{aligned}
$$

where the second term refers to $n_{H_{L}}$ copies of the Higgs bosons $H, H^{c}$ as in Eq. (5), the third for $n_{H_{R}}$ copies of GUT Higgs bosons $H_{R}, H_{R}^{c}$ in $(4,2,1),(\overline{4}, \overline{2}, 1)$ and the last for $n_{D}$ copies of $D$ sextet fields in $(6,1,1)$. These extra fields are necessary in order to guarantee that the gauge couplings remain unified above $M_{U}$ [15]. Running of gauge couplings and gauginos: 
TABLE I. Numerical values of the $Y$ nonrenormalizable operators, described in Eqs. (11)-(14), computed at the unification scale for two choices of $M_{\nu}$.

\begin{tabular}{ccccccc}
\hline \hline$M_{\nu} / M_{U}$ & $Y^{1}$ & $Y^{\text {Ad }}$ & $Y^{B}$ & $Y^{C}$ & $Y^{D}$ & $Y^{33}$ \\
\hline 1.0000 & 0.0110 & -0.00032 & -0.0151 & 0.0056 & 0.0074 & 0.958 \\
0.0001 & 0.0131 & -0.00038 & -0.0194 & 0.0067 & 0.0087 & 1.310 \\
\hline
\end{tabular}

$$
16 \pi^{2} \frac{d g_{i}}{d t}=b_{i} g_{i}^{3}, \quad 16 \pi^{2} \frac{d M_{i}}{d t}=2 b_{i} M_{i} g_{i}^{2}
$$

Running of superpotential Yukawa couplings:

$$
\begin{aligned}
16 \pi^{2} \frac{d \lambda_{u}}{d t}= & \lambda_{u}\left[3 \operatorname{tr}\left\{\lambda_{u}^{\dagger} \lambda_{u}\right\}+\operatorname{tr}\left\{\lambda_{\nu}^{\dagger} \lambda_{\nu}\right\}\right. \\
& \left.+3 \lambda_{u}^{\dagger} \lambda_{u}+\lambda_{d}^{\dagger} \lambda_{d}-2 c_{i}^{\lambda} g_{i}^{2}\right], \\
16 \pi^{2} \frac{d \lambda_{d}}{d t}= & \lambda_{d}\left[3 \operatorname{tr}\left\{\lambda_{d}^{\dagger} \lambda_{d}\right\}+\operatorname{tr}\left\{\lambda_{e}^{\dagger} \lambda_{e}\right\}\right. \\
& \left.+3 \lambda_{d}^{\dagger} \lambda_{d}+\lambda_{u}^{\dagger} \lambda_{u}-2 c_{i}^{\lambda} g_{i}^{2}\right], \\
16 \pi^{2} \frac{d \lambda_{\nu}}{d t}= & \lambda_{\nu}\left[3 \operatorname{tr}\left\{\lambda_{u}^{\dagger} \lambda_{u}\right\}+\operatorname{tr}\left\{\lambda_{\nu}^{\dagger} \lambda_{\nu}\right\}\right. \\
& \left.+3 \lambda_{\nu}^{\dagger} \lambda_{\nu}+\lambda_{e}^{\dagger} \lambda_{e}-2 c_{i}^{\lambda} g_{i}^{2}\right], \\
16 \pi^{2} \frac{d \lambda_{e}}{d t}= & \lambda_{e}\left[3 \operatorname{tr}\left\{\lambda_{d}^{\dagger} \lambda_{d}\right\}+\operatorname{tr}\left\{\lambda_{e}^{\dagger} \lambda_{e}\right\}\right. \\
& \left.+3 \lambda_{e}^{\dagger} \lambda_{e}+\lambda_{\nu}^{\dagger} \lambda_{\nu}-2 c_{i}^{\lambda} g_{i}^{2}\right] .
\end{aligned}
$$

Running of Higgs parameter:

$$
\begin{aligned}
16 \pi^{2} \frac{d \mu}{d t}= & \mu\left[3 \operatorname{tr}\left\{\lambda_{u}^{\dagger} \lambda_{u}\right\}+3 \operatorname{tr}\left\{\lambda_{d}^{\dagger} \lambda_{d}\right\}\right. \\
& \left.+\operatorname{tr}\left\{\lambda_{\nu}^{\dagger} \lambda_{\nu}\right\}+\operatorname{tr}\left\{\lambda_{e}^{\dagger} \lambda_{e}\right\}-2 c_{i}^{2 h} g_{i}^{2}\right] .
\end{aligned}
$$

Running of soft trilinear Yukawa couplings:

$$
\begin{aligned}
16 \pi^{2} \frac{d \tilde{\lambda_{u}}}{d t}= & \tilde{\lambda_{u}}\left[3 \operatorname{tr}\left\{\lambda_{u}^{\dagger} \lambda_{u}\right\}+\operatorname{tr}\left\{\lambda_{\nu}^{\dagger} \lambda_{\nu}\right\}+5 \lambda_{u}^{\dagger} \lambda_{u}\right. \\
& \left.+\lambda_{d}^{\dagger} \lambda_{d}-2 c_{i}^{\lambda} g_{i}^{2}\right]+2 \lambda_{u}\left[3 \operatorname{tr}\left\{\lambda_{u}^{\dagger} \tilde{\lambda_{u}}\right\}+\operatorname{tr}\left\{\lambda_{\nu}^{\dagger} \tilde{\lambda_{\nu}}\right\}\right. \\
& \left.+2 \lambda_{u}^{\dagger} \tilde{\lambda_{u}}+\lambda_{d}^{\dagger} \tilde{\lambda_{d}}+2 c_{i}^{\lambda} M_{i} g_{i}^{2}\right], \\
16 \pi^{2} \frac{d \tilde{\lambda_{d}}}{d t}= & \tilde{\lambda_{d}}\left[3 \operatorname{tr}\left\{\lambda_{d}^{\dagger} \lambda_{d}\right\}+\operatorname{tr}\left\{\lambda_{e}^{\dagger} \lambda_{e}\right\}+5 \lambda_{d}^{\dagger} \lambda_{d}+\lambda_{u}^{\dagger} \lambda_{u}\right. \\
& \left.-2 c_{i}^{\lambda} g_{i}^{2}\right]+2 \lambda_{d}\left[3 \operatorname{tr}\left\{\lambda_{d}^{\dagger} \tilde{\lambda_{d}}\right\}+\operatorname{tr}\left\{\lambda_{e}^{\dagger} \tilde{\lambda_{e}}\right\}+2 \lambda_{d}^{\dagger} \tilde{\lambda_{d}}\right. \\
& \left.+\lambda_{u}^{\dagger} \tilde{\lambda_{u}}+2 c_{i}^{\lambda} M_{i} g_{i}^{2}\right],
\end{aligned}
$$

TABLE II. Numerical values of the most stable quark $\left(V^{Q}\right)$ and lepton $\left(V^{L}\right)$ mixing angles computed to best fit the CKM matrix using selected operators and texture adopted in Eqs. (11)-(14) (see also Table III).

\begin{tabular}{ccccc}
\hline \hline & \multicolumn{2}{c}{$V^{Q}$} & \multicolumn{2}{c}{$V^{L}$} \\
& Weak & GUT & Weak & GUT \\
\hline$V_{12}$ & 0.2210 & 0.2210 & 0.0625 & 0.0625 \\
$V_{11}$ & 0.9752 & 0.9752 & 0.9980 & 0.9980 \\
$V_{22}$ & 0.9743 & 0.9747 & 0.9974 & 0.9976 \\
$V_{33}$ & 0.9990 & 0.9995 & 0.9993 & 0.9995 \\
\hline \hline
\end{tabular}

$$
\begin{aligned}
16 \pi^{2} \frac{d \tilde{\lambda_{\nu}}}{d t}= & \tilde{\lambda}_{\nu}\left[3 \operatorname{tr}\left\{\lambda_{u}^{\dagger} \lambda_{u}\right\}+\operatorname{tr}\left\{\lambda_{\nu}^{\dagger} \lambda_{\nu}\right\}+5 \lambda_{\nu}^{\dagger} \lambda_{\nu}+\lambda_{e}^{\dagger} \lambda_{e}\right. \\
& \left.-2 c_{i}^{\lambda} g_{i}^{2}\right]+2 \lambda_{\nu}\left[3 \operatorname{tr}\left\{\lambda_{u}^{\dagger} \tilde{\lambda_{u}}\right\}+\operatorname{tr}\left\{\lambda_{\nu}^{\dagger} \tilde{\lambda_{\nu}}\right\}+2 \lambda_{\nu}^{\dagger} \tilde{\lambda_{\nu}}\right. \\
& \left.+\lambda_{e}^{\dagger} \tilde{\lambda_{e}}+2 c_{i}^{\lambda} M_{i} g_{i}^{2}\right], \\
16 \pi^{2} \frac{d \tilde{\lambda_{e}}}{d t}= & \tilde{\lambda_{e}}\left[3 \operatorname{tr}\left\{\lambda_{d}^{\dagger} \lambda_{d}\right\}+\operatorname{tr}\left\{\lambda_{e}^{\dagger} \lambda_{e}\right\}+5 \lambda_{e}^{\dagger} \lambda_{e}+\lambda_{\nu}^{\dagger} \lambda_{\nu}\right. \\
& \left.-2 c_{i}^{\lambda} g_{i}^{2}\right]+2 \lambda_{e}\left[3 \operatorname{tr}\left\{\lambda_{d}^{\dagger} \tilde{\lambda_{d}}\right\}+\operatorname{tr}\left\{\lambda_{e}^{\dagger} \tilde{\lambda_{e}}\right\}+2 \lambda_{e}^{\dagger} \tilde{\lambda_{e}}\right. \\
& \left.+\lambda_{\nu}^{\dagger} \tilde{\lambda_{\nu}}+2 c_{i}^{\lambda} M_{i} g_{i}^{2}\right] .
\end{aligned}
$$

Running of soft Higgs parameter:

$$
\begin{aligned}
16 \pi^{2} \frac{d \tilde{\mu}^{2}}{d t}= & \tilde{\mu}^{2}\left[3 \operatorname{tr}\left\{\lambda_{u}^{\dagger} \lambda_{u}\right\}+3 \operatorname{tr}\left\{\lambda_{d}^{\dagger} \lambda_{d}\right\}\right. \\
& \left.+\operatorname{tr}\left\{\lambda_{\nu}^{\dagger} \lambda_{\nu}\right\}+\operatorname{tr}\left\{\lambda_{e}^{\dagger} \lambda_{e}\right\}-2 c_{i}^{2 h} g_{i}^{2}\right] \\
& +2 \mu\left[3 \operatorname{tr}\left\{\lambda_{u}^{\dagger} \widetilde{\lambda_{u}}\right\}+3 \operatorname{tr}\left\{\lambda_{d}^{\dagger} \tilde{\lambda_{d}}\right\}+\operatorname{tr}\left\{\lambda_{\nu}^{\dagger} \tilde{\lambda_{\nu}}\right\}\right. \\
& \left.+\operatorname{tr}\left\{\lambda_{e}^{\dagger} \tilde{\lambda_{e}}\right\}+2 c_{i}^{2 h} M_{i} g_{i}^{2}\right] .
\end{aligned}
$$

Running of soft scalar masses:

$$
\begin{aligned}
& 16 \pi^{2} \frac{d \tilde{m}_{Q}^{2}}{d t}= {\left[\tilde{m}_{Q}^{2} \lambda_{u}^{\dagger} \lambda_{u}+\lambda_{u}^{\dagger}\left(m_{H_{u}}^{2}+\tilde{m}_{u^{c}}^{2}\right) \lambda_{u}+\tilde{\lambda}_{u}^{\dagger} \tilde{\lambda_{u}}+\tilde{m}_{Q}^{2} \lambda_{d}^{\dagger} \lambda_{d}\right.} \\
&\left.+\lambda_{d}^{\dagger}\left(m_{H_{d}}^{2}+\tilde{m}_{d^{c}}^{2}\right) \lambda_{d}+\tilde{\lambda}_{d}^{\dagger} \tilde{\lambda_{d}}+\text { H.c. }\right]-8 c_{i}^{F} M_{i}^{2} g_{i}^{2}, \\
& 16 \pi^{2} \frac{d \tilde{m}_{u^{c}}^{2}}{d t}=2\left[\tilde{m}_{u^{c}}^{2} \lambda_{u} \lambda_{u}^{\dagger}+\lambda_{u}\left(m_{H_{u}}^{2}+\tilde{m}_{Q}^{2}\right) \lambda_{u}^{\dagger}+\tilde{\lambda_{u}}{\tilde{\lambda_{u}^{\dagger}}}^{\dagger}+\text { H.c. }\right] \\
&-8 c_{i}^{F^{c}} M_{i}^{2} g_{i}^{2}, \\
& 16 \pi^{2} \frac{d \tilde{m}_{d^{c}}^{2}=}{d t}=2\left[\tilde{m}_{d^{c}}^{2} \lambda_{d} \lambda_{d}^{\dagger}+\lambda_{d}\left(m_{H_{d}}^{2}+\tilde{m}_{Q}^{2}\right) \lambda_{d}^{\dagger}+\tilde{\lambda_{d}} \tilde{\lambda}_{d}^{\dagger}+\text { H.c. }\right] \\
&-8 c_{i}^{F^{c}} M_{i}^{2} g_{i}^{2},
\end{aligned}
$$




$$
\widetilde{S}^{l} \widetilde{M}^{l 2} \widetilde{S}^{l \dagger}=\tilde{M}^{l 2}(d), \quad \tilde{M}^{l 2}=\left(\begin{array}{cc}
\tilde{M}_{L L}^{l 2} & \tilde{M}_{L R}^{l 2} \\
\tilde{M}_{R L}^{l 2} & \tilde{M}_{R R}^{l 2}
\end{array}\right), \quad \widetilde{S}^{l}=\left(\begin{array}{cc}
\widetilde{S}_{L L}^{l} & \widetilde{S}_{L R}^{l} \\
\widetilde{S}_{R L}^{l} & \widetilde{S}_{R R}^{l}
\end{array}\right),
$$

and has eigenvalues denoted by $\tilde{m}_{l}^{2}\left(\tilde{m}_{l_{A}}^{2}=\tilde{m}_{l_{A_{L}}}^{2}, \tilde{m}_{l_{A+3}}^{2}=\tilde{m}_{l_{\tilde{A}}}^{2}=\tilde{m}_{l_{A_{R}}}^{2}\right)$.

The left-handed $12 \times 12$ sneutrino mass matrix in the basis $\phi=\left(\phi_{L}, \phi_{R}\right)$, where $\phi_{L}=\left(\tilde{\nu}, \tilde{\nu}^{*}\right)$ and $\phi_{R}=\left(\tilde{\nu}^{c}, \tilde{\nu}^{c *}\right)$ is given by

$$
\tilde{M}^{\phi 2}=\left(\begin{array}{cc}
\tilde{M}_{L L}^{\phi 2} & \tilde{M}_{L R}^{\phi 2} \\
\widetilde{M}_{L L}^{\phi 2} & \tilde{M}_{R R}^{\phi 2}
\end{array}\right)
$$

with the $6 \times 6$ blocks defined as

$$
\begin{aligned}
& \tilde{M}_{L L}^{\phi 2}=1 / 2\left(\begin{array}{cc}
\tilde{m}_{L}^{2}+m_{\nu}^{\dagger} m_{\nu}+m_{Z}^{2} Z_{\nu} c_{2 \beta} & 0 \\
0 & \tilde{m}_{L}^{2}+m_{\nu}^{T} m_{\nu}^{*}+m_{Z}^{2} Z_{\nu} c_{2 \beta}
\end{array}\right), \\
& \tilde{M}_{R R}^{\phi 2}=1 / 2\left(\begin{array}{cc}
\tilde{m}_{\nu^{c}}^{2}+m_{\nu}^{*} m_{\nu}^{T}+M_{\nu}^{\dagger} M_{\nu} & \tilde{M}_{\nu}^{2 \dagger} \\
\tilde{M}_{\nu}^{2} & \tilde{m}_{\nu^{c}}^{2}+m_{\nu} m_{\nu}^{\dagger}+M_{\nu}^{T} M_{\nu}^{*}
\end{array}\right), \\
& \tilde{M}_{L R}^{\phi 2}=1 / 2\left(\begin{array}{cc}
m_{\nu}^{\dagger} M_{\nu} & v_{u} \tilde{\Lambda}_{\nu}^{\dagger}-\mu v_{d} \lambda_{\nu}^{\dagger} \\
v_{u} \tilde{\Lambda}_{\nu}^{T}-\mu v_{d} \lambda_{\nu}^{T} & m_{\nu}^{T} M_{\nu}^{*}
\end{array}\right),
\end{aligned}
$$

and $\widetilde{M}_{R L}^{\phi 2}=\widetilde{M}_{L R}^{\phi 2 \dagger}$. In general, to find the eigenvalues of $\widetilde{M}^{\phi 2}$ we need to diagonalize the full sneutrino matrix in Eq. (C9). However, since this matrix depends on two very distinct scales, the electro-weak VEVs and the right-hand $M_{\nu}$ mass, the lightest sneutrinos are approximately described by an effective $6 \times 6$ block matrix given by

$$
\begin{aligned}
\left(\widetilde{M}_{L L}^{\phi 2}\right)^{\text {light }} & =\widetilde{M}_{L L}^{\phi 2}-\widetilde{M}_{L R}^{\phi 2} \times\left(\widetilde{M}_{R R}^{\phi 2}\right)^{-1} \times \widetilde{M}_{R L}^{\phi 2} \\
& =1 / 2\left(\begin{array}{cc}
\tilde{m}_{L}^{2}+m_{Z}^{2} Z_{\nu} c_{2 \beta} & 0 \\
0 & \tilde{m}_{L}^{2}+m_{Z}^{2} Z_{\nu} c_{2 \beta}
\end{array}\right) .
\end{aligned}
$$

Comparing Eqs. (C10) and (C13) we observe that, up to very suppressed terms of order $m_{\nu} / M_{\nu}$, the two matrices are equal except for the Dirac mass contribution $m_{\nu}^{2}$ absent in $\left(\widetilde{M}_{L L}^{\phi 2}\right)^{\text {light. }}$ Therefore, the light sneutrinos $\phi_{L}^{\text {light }} \sim \phi_{L}$ have masses

$$
\phi_{L}^{T}\left(\tilde{M}_{L L}^{\phi 2}\right)^{\text {light }} \phi_{L} \sim \tilde{\nu}^{*}\left(\tilde{m}_{L}^{2}+m_{Z}^{2} Z_{\nu} c_{2 \beta}\right) \tilde{\nu}=\tilde{\nu}^{*} \tilde{M}_{L L}^{n 2} \tilde{\nu} .
$$

The matrix $\widetilde{M}_{L L}^{n 2}$ is diagonalized by $\widetilde{S}_{L L}^{n}$ defined through

$$
\widetilde{S}_{L L}^{n} \widetilde{M}_{L L}^{n 2} \widetilde{S}_{L L}^{n \dagger}=\widetilde{M}^{n 2}(d),
$$

and has eigenvalues $\tilde{m}_{n}^{2}\left(\tilde{m}_{n_{A}}^{2}=\tilde{m}_{n_{A_{L}}}^{2}, \tilde{m}_{n_{A+3}}^{2}=\tilde{m}_{n_{\tilde{A}}}^{2}=\tilde{m}_{n_{A_{R}}}^{2}\right)$. The $\widetilde{U} \mathrm{~s}$ in Eqs. (23)-(25) are defined by

$$
\begin{gathered}
\widetilde{U}_{L L}^{n}=\widetilde{S}_{L L}^{n} T^{e^{\dagger}} \\
\widetilde{U}^{l}=\left(\begin{array}{cc}
\widetilde{U}_{L L}^{l} & \widetilde{U}_{L R}^{l} \\
\widetilde{U}_{R L}^{l} & \widetilde{U}_{R R}^{l}
\end{array}\right)=\left(\begin{array}{cc}
\widetilde{S}_{L L}^{l} T^{e \dagger} & \widetilde{S}_{L R}^{l} S^{e^{\dagger}} \\
\widetilde{S}_{R L}^{l} T^{e \dagger} & \widetilde{S}_{R R}^{l} S^{e^{\dagger}}
\end{array}\right) .
\end{gathered}
$$

Finally we provide the expressions for $J$ and $H$ appearing in Eqs. (23)-(25):

$$
\begin{gathered}
J_{i j A}=\sum_{k=1,2}\left(g_{1} S_{i k}^{C \dagger}\right)\left(g_{1} T_{k j}^{C}\right) m_{C_{k}} / \tilde{m}_{n_{A}}^{2} J_{k A}, \\
H_{p q A}=\sum_{r=1 \ldots 4}\left(g_{p} S_{p r}^{N \dagger}\right)\left(g_{q} S_{r q}^{N}\right) m_{N_{r}} / \tilde{m}_{l_{A}}^{2} H_{r A}, \\
J_{k A}=\mathcal{J}\left(m_{C_{k}}^{2} / \tilde{m}_{n_{A}}^{2}\right), \quad H_{r A}=\mathcal{H}\left(m_{N_{r}}^{2} / \tilde{m}_{l_{A}}^{2}\right) .
\end{gathered}
$$

Here $g_{p, q}=\left(g^{\prime}, g, g, g\right)$ and the function $\mathcal{J}(\mathcal{H})$ arises from chargino (neutralino) loop integration [6]. The (supersymmetric state) indices $i, j, p$ and $q$ can take values among $i$ $=(1,2)=\left(\widetilde{W}_{L}^{-}, \widetilde{H}_{L}^{-}\right), \quad j=(1,2)=\left(\widetilde{W}_{R}^{-}, \widetilde{H}_{R}^{-}\right), \quad p \quad$ and $\quad q$ $=(1,2,3,4)=\left(\widetilde{B}, \widetilde{W}^{0}, \widetilde{H}_{d}^{0}, \widetilde{H}_{u}^{0}\right) . \quad S^{C}$ and $T^{C}$ diagonalize $\left[S^{C} M^{C} T^{C \dagger}=M^{C}(d)\right]$ the $2 \times 2$ chargino mass matrix $M^{C}$ which has eigenvalues $m_{C_{k}}$. Similarly $S^{N}$ diagonalizes $\left[S^{N} M^{N} S^{N \dagger}=M^{N}(d)\right]$ the $4 \times 4$ neutralino mass matrix $M^{N}$ which has eigenvalues $m_{N_{r}}$ : 


$$
\begin{aligned}
M^{C} & =\left(\begin{array}{ccc}
M_{2} & \sqrt{2} m_{W} s_{\beta} \\
\sqrt{2} m_{W} c_{\beta} & \mu
\end{array}\right), \\
M^{N} & =\left(\begin{array}{cccc}
M_{1} & 0 & -m_{Z} c_{\beta} s_{\theta} & m_{Z} s_{\beta} s_{\theta} \\
0 & M_{2} & m_{Z} c_{\beta} c_{\theta} & -m_{Z} s_{\beta} c_{\theta} \\
-m_{Z} c_{\beta} s_{\theta} & m_{Z} c_{\beta} c_{\theta} & 0 & -\mu \\
m_{Z} s_{\beta} s_{\theta} & -m_{Z} s_{\beta} c_{\theta} & -\mu & 0
\end{array}\right) .
\end{aligned}
$$

APPENDIX D: FERMION MASSES AND MIXING ANGLES

This appendix is intended to give an overview of fermion masses and mixing angles predicted by this model. Ultimately we have in mind their comparison with the available data. We will focus on the effects associated with the running of the parameters between the weak and GUT scale and their variation with the right-handed neutrino decoupling scale $M_{\nu}$. For convenience we show below the CKM matrix [23]:

$$
V^{Q}=\left(\begin{array}{ccc}
0.9747-0.9759 & 0.218-0.224 & 0.002-0.007 \\
0.218-0.224 & 0.9735-0.9751 & 0.032-0.054 \\
0.003-0.018 & 0.030-0.054 & 0.9985-0.9995
\end{array}\right)
$$

The results in Tables I-IV correspond to input values of $\alpha_{s}=0.115$ and $m_{\text {bottom }}=4.25 \mathrm{GeV}$. Variations due to different choices of these parameters are significant ${ }^{9}$ and have been partially considered in Ref. [18]. Since we worked with one-loop RGE all the tables are independent of the Planck scale parameters $m_{0}, M_{1 / 2}$ and $A_{0}$.

In Table I, we show the values that the $Y$ operators appearing in Eqs. (11)-(14) take at the GUT scale, for two values of $M_{\nu}=M_{U}$ and $M_{\nu}=2 \times 10^{12} \mathrm{GeV}$. Operators B and 33 can be seen to be the most sensitive to $M_{\nu}$.

In Table II, we have collected the mixing angles which are approximately insensitive to changes in $M_{\nu}$ and stable relative to RGE effects. We denoted the quark CKM matrix by $V^{Q}$ and the leptonic counterpart by $V^{L}\left(V_{21} \sim V_{12}\right)$. The Clebsch factors in Eqs. (11)-(14) imply ${ }^{10} V_{12}^{L} \sim V_{12}^{Q} / 4$.

In Table III we include the remaining $V^{Q}$ and $V^{L}$ entries

\footnotetext{
${ }^{9}$ See for example Fig. 1.

${ }^{10}$ It is relevant to note that the $Y$ operators were chosen because they can not only account for the experimental fermion mass pattern but predict successfully "natural" mixing angles as well. By this we mean that we were careful to select them in such a way that none arises as the residue of an almost complete cancellation of the contributions coming from the up and down Yukawa matrices.
}

not present in Table II $\left(M_{\nu}=2 \times 10^{16} \mathrm{GeV}\right)$. The reason for the discrimination is threefold. First because we are now confronted with values that are more sensitive to variations in $M_{\nu}$. For example, taking $M_{\nu}=10^{-4} \times M_{U}$ effects the values shown to about $7 \%$. Secondly because the mixings in Table III are generally not as stable to RGE effects as the ones in Table II. And finally because we wanted to call attention to the fact that the values that are actually used when we computed LFV processes, denoted by output, are not exactly the same as the ones we have available from experiment (input). The discrepancy arises when we replace the GUT Yukawa couplings by others parametrized by our set of operators arranged in a successful "texture."

Finally in Table IV, we present the predictions for some fermion masses. Whenever two values are shown for the same parameter, the first is associated with $M_{\nu}=10^{-4}$ $\times M_{U}$ while the second with $M_{\nu}=M_{U}$. The down (strange) quark has a mass within the 5-15 (100-300) MeV range quoted in Ref. [23]. The $m_{\nu}$ values correspond to the unphysical mass directly obtained from the neutrino Yukawa couplings [for example $m_{\nu_{\tau}} \sim v_{u}\left(\lambda_{\nu}\right)_{\tau \tau}$ ]. On the other hand, the physical mass of the tau-neutrino is correctly obtained after taking into account the see-saw suppression mechanism which forces it to scale as $m_{\nu_{\tau}}^{2} / 4 M_{\nu}$. In all cases we obtained predictions fairly compatible with experimental data.
[1] R. Barbieri and L. J. Hall, Phys. Lett. 33B, 212 (1994).

[2] R. Barbieri, L. J. Hall, and A. Strumia, Nucl. Phys. B445, 219 (1995)

[3] F. Borzumati and A. Masiero, Phys. Rev. Lett. 57, 961 (1986); L. J. Hall, V. A. Kostelecky, and S. Raby, Nucl. Phys. B267,
415 (1986); T. Kosmas, G. K. Leontaris, and J. D. Vergados, Phys. Lett. B 219, 457 (1989).

[4] E. Cremmer, S. Ferrara, and J. Scherk, Phys. Lett. 74B, 61 (1978); H. P. Nilles, Phys. Rep. 110, 1 (1984).

[5] V. Barger, M. S. Berger, P. Ohmann, and R. J. N. Philips, 
hep-ph/9308233; P. Langacker and N. Polonsky, Phys. Rev. D 49, 1454 (1994); G. Kane, C. Kolda, L. Roszkowski, and J. Wells, ibid. 49, 6173 (1994); N. Polonsky and A. Pomarol, ibid. 51, 6532 (1995).

[6] J. Hisano, T. Moroi, K. Tobe, and M. Yamaguchi, Phys. Rev. D 53, 2442 (1996).

[7] S. F. King and Q. Shafi, Phys. Lett. B 422, 135 (1998).

[8] J. C. Pati and A. Salam, Phys. Rev. D 10, 275 (1974).

[9] I. Antoniadis and G. K. Leontaris, Phys. Lett. B 216, 333 (1989); I. Antoniadis, G. K. Leontaris, and J. Rizos, ibid. 245, 161 (1990).

[10] B. C. Allanach and S. F. King, Nucl. Phys. B459, 75 (1996).

[11] N. Arkani-Hamed, H. Cheng, and L. J. Hall, Phys. Rev. D 53, 413 (1996).

[12] G. K. Leontaris and N. D. Tracas, Phys. Lett. B 419, 206 (1998); G. K. Leontaris and N. D. Tracas, ibid. 431, 90 (1998).

[13] H. Arason, D. J. Castano, E. J. Piard, and P. Ramond, Phys. Rev. D 47, 232 (1993).

[14] V. Barger, M. S. Berger, and P. Ohmann, Phys. Rev. D 47, 1093 (1993).

[15] S. F. King, Phys. Lett. B 325, 129 (1994).

[16] G. Anderson, S. Dimopoulos, L. J. Hall, S. Raby, and G. D. Starkman, Phys. Rev. D 49, 3660 (1994).

[17] H. Georgi and C. Jarlskog, Phys. Lett. 89B, 297 (1978); P. Ramond, R. G. Roberts, and G. G. Ross, Nucl. Phys. B406, 19 (1993); L. J. Hall and A. Rasin, Phys. Lett. B 315, 164 (1993).
[18] B. C. Allanach and S. F. King, Nucl. Phys. B456, 57 (1995).

[19] B. C. Allanach and S. F. King, Phys. Lett. B 353, 477 (1995).

[20] S. P. Martin and M. T. Vaughn, Phys. Rev. D 50, 2282 (1994).

[21] B. C. Allanach and S. F. King, Phys. Lett. B 328, 360 (1994).

[22] S. G. Gorshny, A. L. Kataev, and S. A. Larin, Phys. Lett. 135B, 457 (1984); O. V. Tarasov, A. A. Vladimirov, and A. Y. Zharkov, ibid. 93B, 429 (1980).

[23] Particle Data Book, R. M. Barnett et al., Phys. Rev. D 54, 1 (1996), p. 58.

[24] ALEPH Collaboration, R. Barate et al., Eur. Phys. J. C 2, 417 (1998).

[25] DELPHI Collaboration, P. Abreu et al., Eur. Phys. J. C 1, 1 (1998).

[26] L3 Collaboration, M. Acciarri et al., Z. Phys. C: Part Fields 4, 207 (1995).

[27] OPAL Collaboration, K. Ackerstaff et al., Eur. Phys. J. C 2, 213 (1998).

[28] OPAL Collaboration, OPAL LEPC Presentation, D. Plane (1998).

[29] ALEPH Collaboration, ALEPH Status Report to LEPC, E. Lancon (1998).

[30] DELPHI Collaboration, DELPHI Status Report to LEPC, V. Ruhlmann-Kleider (1998).

[31] M. E. Gómez, G. K. Leontaris, S. Lola, and J. D. Vergados, Phys. Rev. D 59, 116009 (1999). 